\title{
Ensemble Data Assimilation in the Red Sea: Sensitivity to Ensemble Selection and Atmospheric Forcing
}

Habib Toye ${ }^{1}$, Peng Zhan², Ganesh Gapalakrishnan³ ${ }^{3}$ Aditya R. Kartadikaria' ${ }^{2,4}$, Huang Huang ${ }^{1}$, Omar Knio ${ }^{1}$, and Ibrahim Hoteit ${ }^{1,2, *}$

${ }^{1}$ Division of Computer, Electrical and Mathematical Sciences and Engineering, King Abdullah University of Science and Technology (KAUST), Thuwal, Saudi Arabia 2Division of Physical Sciences and Engineering, King Abdullah University of Science and Technology (KAUST), Thuwal, Saudi Arabia ${ }^{3}$ Scripps Institution of Oceanography, University of California San Diego, La Jolla, California, USA

${ }^{4}$ Study Program of Oceanography, Bandung Institute of Technology, Bandung, Indonesia

*Correspondence to: I. Hoteit, ibrahim.hoteit@kaust.edu.sa 


\begin{abstract}
We present our efforts to build an ensemble data assimilation and forecasting system for the Red Sea. The system consists of the high-resolution Massachusetts Institute of Technology general circulation model (MITgcm) to simulate ocean circulation and of the Data Research Testbed (DART) for ensemble data assimilation. DART has been configured to integrate all members of an ensemble adjustment Kalman filter (EAKF) in parallel, based on which we adapted the ensemble operations in DART to use an invariant ensemble, i.e. an ensemble Optimal Interpolation (EnOI) algorithm. This approach requires only single forward model integration in the forecast step, and therefore saves substantial computational cost. To deal with the strong seasonal variability of the Red Sea, the EnOI ensemble is then seasonally selected from a climatology of long-term model outputs. Observations of remote sensing sea surface height (SSH) and sea surface temperature (SST) are assimilated every three days. Real time atmospheric fields from the National Center for Environmental Prediction (NCEP) and the European Centre for Medium-Range Weather Forecasts (ECMWF) are used as forcing in different assimilation experiments. We investigate the behaviors of the EAKF and (seasonal-) EnOI and compare their performances for assimilating and forecasting the circulation of the Red Sea. We further assess the sensitivity of the assimilation system to various filtering parameters (ensemble size, inflation) and atmospheric forcing.
\end{abstract}

Keywords Red Sea, Data Assimilation, Seasonal Variability, Ensemble Kalman Filter, Ensemble Optimal Interpolation. 


\section{Introduction}

The Red Sea is one of the most important world marginal seas, in terms of its genetic variation and biodiversity with endemic species of fish, its ecological importance with the second largest community of coral reefs, its economic impact on the surrounding countries' development and on the international trade route, and so forth. The Red Sea has a nearly enclosed basin dividing Asia from Africa. The basin is about $2000 \mathrm{~km}$ long and less than $300 \mathrm{~km}$ wide, with a deep trench along its axis and extensive shallow shelves on both shores supporting a rich ecosystem. The only significant connection between the Red Sea and the open (Indian) ocean is the Strait of Bab el Mandeb, through which a remarkable seasonally varying water exchange has been well documented and studied through in-situ observations (Johns and Sofianos 2012; Murray and Johns 1997) and numerical modeling (Yao et al. 2014a; Yao et al. 2014b). The Red Sea circulation is highly dynamic and is characterized by basin-scale overturning circulation, strong boundary currents, and multiple transient and recurrent mesoscale eddies. Subject to the Indian Monsoon, the Red Sea circulation exhibits a seasonal variability, which involves annual cycles of water exchange with the Gulf of Aden, the overturning circulation (Yao et al. 2014a; Yao et al. 2014b), and the intensity of eddy activity (Zhan et al. 2014; Zhan et al. 2016).

Because of the sporadic coverage of the observations in the Red Sea, most basinscale studies have been reported based on numerical simulations, either using a simplified model (Tragou and Garrett 1997; Zhai et al. 2015; Zhai and Marshall 2013), a climatology of general circulation model (GCM) outputs (Eshel and Naik 1997; Siddall et al. 2002; Sofianos and Johns 2002; Sofianos and Johns 2003), or an ocean general circulation model forced with real atmospheric conditions (Chen et al. 2014; Triantafyllou et al. 2014; Yao et al. 2014a; Yao et al. 2014b; Zhan et al. 2016). Ocean models are however not perfect and can be subject to many sources of uncertainties. These may come from inaccurate atmospheric forcing, initial and boundary conditions, bathymetry, modeling and numerical errors, and poorly known parameters. This should not question the ability of these models to provide a 
general dynamical picture of the ocean circulation of the domain of interest, especially at large scales, but it would limit their ability to provide accurate forecasts for planning and decision-making. Data assimilation is nowadays recognized as the most efficient approach to develop reliable ocean operational systems. The Red Sea is extremely lagging behind in this context, with the only data assimilation study backdating to the mid-nineties by Clifford et al. (1997). In that study, the authors tested a nudging-based assimilation system the simplest form of assimilation, for the Red Sea, using a 6-7 km resolution model forced by simplified atmospheric fields to assimilate sea surface temperature (SST) and some profile data measured by XBT/CTD. The availability of remote sensing ocean data, which have been validated and used to study the Red Sea (Zhai and Bower 2013; Zhan et al. 2014), is yet to be exploited for assimilation. A state-of-the-art modeling system with data assimilation in this region should improve the predictability of the Red Sea circulation and its strong eddy activity, and setup the infrastructure for developing a Red Sea operational system.

One of thrusts of the recently established Saudi Aramco Marine Environmental Research Center at KAUST (SAMERCK) is to establish an operational oceanography for the Red Sea (https://iop.kaust.edu.sa). The comprehensive and realistic outputs of ocean forecasts would benefit not only the booming industrial developments and shipping activities in the Red Sea, but would also provide more realistic simulations needed for the fundamental understanding of the Red Sea general circulation and eddy activities. A Red Sea operational system is greatly needed for predicting circulation and transport phenomena, including oil spills, pollutants and nutrients. Such a system will be further very helpful for supporting various scientific activities, ecosystem conservation, aquaculture activities, search-and-rescue, shipping industry, Navy operations, etc. As part of the ongoing phase-1 of the SAMERCK project, a pre-operational ensemble data assimilation system is being developed that is capable of assimilating any available observations in the Red Sea. Our first goal is to implement an efficient, in term of computational cost and performances, 
ensemble-based assimilation system with a high-resolution general circulation ocean model of the Red Sea and to test it for assimilation of remote sensing data.

In this work, we examine the overall performance of a deterministic ensemble Kalman filter (EnKF), the Ensemble Adjustment Kalman filter (EAKF) (Anderson 2001), for assimilating satellite sea surface height (SSH) and SST data into a $4 \mathrm{~km}$ Massachusetts Institute of Technology general circulation ocean model (MITgcm) that has been configured and validated to study the circulation of the whole Red Sea (Yao et al. 2014a; Yao et al. 2014b; Zhan et al. 2016). We evaluate the sensitivity of this assimilation system to various parameters and inputs, including filtering scheme and parameters (ensemble size, inflation) and atmospheric fields (NCEP and ECMWF). We are in particular interested in investigating the benefit of integrating the filter ensemble with the dynamical model (i.e. using a flow-dependent ensemble) against keeping it invariant in time, using an Ensemble Optimal Interpolation (EnOI)-like scheme in which the model is used to forecast only the state, and not the ensemble (Fu et al. 2011; Hoteit et al. 2002; Oke et al. 2007; Sakov and Sandery 2015). The latter assumes that the forecast error covariance is well represented by a stationary ensemble, and may lead to drastic reduction (up to 80$90 \%$ less) in the computational burden compared to a flow-dependent ensemble. It may further help maintaining the ensemble spread, which is one of the issues often encountered in EAKF applications, especially when model errors are not directly accounted for in the system (Anderson 2003; Hoteit et al. 2002). We further assess the possibility of exploiting the dominant seasonal variability of the Red Sea and test the performance of the EnOI scheme with seasonally varying ensemble of model states that are not integrated with ocean GCM, but are readily available from a historical model run (Backeberg et al. 2014; Xie and Zhu 2010). This allows the EnOI scheme to adjust to the seasonal variation of the system without extra computational cost, but may not well-represent the error-of-the day in the most recent estimate compared to an ensemble Kalman filter. We compare the performances of the EAKF, EnOI, and EnOI with seasonal varying ensemble (SEnOI) 
in the Red Sea and study their sensitivities to various settings and atmospheric forcing.

The rest of this paper is organized as follows. Section 2 gives a brief description of the model and observational data used for model validation and in the assimilation experiments. Details of the assimilation schemes and their implementation are provided in Section 3. In Section 4, we present the results of several assimilation experiments that have been conducted to evaluate the performances and robustness of the different ensemble assimilation schemes. A discussion and summary conclude the work in Section 5.

\section{Model and Data}

\subsection{Ocean model and configuration}

The MITgcm is integrated on a $0.04^{\circ} \times 0.04^{\circ}$ spherical polar grid, with 50 vertical z layers ranging from $4 \mathrm{~m}$ at the surface to $300 \mathrm{~m}$ near the bottom. The model domain extends from $30^{\circ} \mathrm{E}$ to $50^{\circ} \mathrm{E}$ and from $10^{\circ} \mathrm{N}$ to $50^{\circ} \mathrm{N}$, covering the Red Sea, the Gulf of Suez, the Gulf of Aqaba and the Gulf of Aden. The bathymetry is extracted from the gridded General Bathymetric Chart of the Ocean (GEBCO, available at http://www.gebco.net/data_and_products/gridded_bathymetry_data/). The model is configured in hydrostatic mode with an implicit free surface. The K-profile parameterization is used for the vertical mixing scheme. The lateral boundaries are treated with no-slip conditions and a quadratic bottom friction is imposed. Zonal and meridional velocities, temperature and salinity from the Estimation of the Circulation and Climate of the Ocean (ECCO) project (Kohl and Stammer 2008) are prescribed as the open boundary conditions in the Gulf of Aden through a 20-km buffer zone. In different experiments, the model is forced with 6-hourly atmospheric reanalysis from National Centers for Environmental Prediction (NCEP) or the European Centre for Medium-Range Weather Forecasts (ECMWF). These include zonal and meridional wind speed, air temperature, specific humidity, precipitation and downward short and long wave heat fluxes. A free model run was integrated over a 32-year period from January 1979 to December 2011 using a time step of 
200s (without assimilation), and outputs from 1992 to 2011 were stored for validation and for constructing an (initial or static) ensemble of state realizations.

\subsection{Observational data}

For assimilation, along-track SSH data is obtained by combining sea level anomaly (SLA) acquired from the Radar Altimeter Database System (RADS available through the web portal http://rads.tudelft.nl) and the mean dynamic topography (MDT) from AVISO (available at ftp.aviso.altimetry.fr/auxiliary/). RADS is developed by Delft University of Technology and National Oceanic and Atmospheric Administration (NOAA). It provides merged SLA observations from nine altimeter missions, and is one of the most accurate and complete data bases of satellite radar altimeter data (Scharroo et al. 2013). MDT is a key reference surface for altimeter data, and can be used to calculate the corresponding absolute dynamic topography (ADT) from the altimeter SLA through ADT = MDT + SLA. The ADT is equivalent to the model SSH, which will be assimilated into the ocean model. All the SSH data within the assimilation window, 3 days in this study, were gathered and assimilated once at the middle of the window. Observational errors of these along-track SSH data are specified with different values ranging between 0.05 to $0.1 \mathrm{~m}$ for different satellite missions.

It is important to point out that the accuracy of altimetry data in coastal waters could be limited by several factors, including the weaknesses of the altimeters in the range tracking procedure close to the shorelines, intrinsic difficulties in the corrections of the wet tropospheric correction, tides, etc., and issues of land contamination in the altimeter return waveforms (Cipollini et al. 2010; Vignudelli et al. 2011). Important efforts are still being carried out to overcome such problems and to extend the capabilities of current and future altimetry data in coastal waters (Le Henaff et al. 2011; Madsen et al. 2015; Tseng et al. 2014; Vignudelli et al. 2011; Yang et al. 2012). As a safe and practical approach, the SSH observations over shallow waters (less than $60 \mathrm{~m}$ in depth) were excluded. We have also removed the outliers during the assimilation process, which were flagged when the 
corresponding prediction error exceeded three times the variance of the prescribed observational error.

The assimilated SST data is extracted from the Group for High Resolution Sea Surface Temperature (GHRSST) global Level 4 SST analysis produced daily on a $1 / 4^{\circ}$ grid at the NOAA National Climatic Data Center (Reynolds et al. 2007) (available at http://podaac.jpl.nasa.gov/dataset/NCDC-L4LRblend-GLOB-AVHRR_OI). These are mapped data from the $4 \mathrm{~km}$ Advanced Very High Resolution Radiometer (AVHRR) Pathfinder Version 5 time series (when available, otherwise operational NOAA AVHRR data are used) and in-situ observations. In the assimilation experiments, three-day averaged data is provided at midnight with a $0.25^{\circ}$ grid. Observational errors are uniform and set at $1.2^{\circ} \mathrm{C}$. These are larger than what is commonly used, but are expected to also account for the spatially correlated nature of this mapped dataset.

\subsection{Model validation with SSH/SST}

A free model run (without assimilation) was integrated over a 32-year period from 1979 to 2011, and its outputs from 1992 to 2011 were stored for validation and for constructing an (initial or static) ensemble of state realizations. As shown in Fig. 1, the model mean SST (Fig. 1.e) is in good agreement with the AVHRR data (Fig. 1.a), exhibiting a clear gradient throughout the basin where highest temperature is found on both coasts of the southern Red Sea. Strong variability of SST near the west coast around $16^{\circ} \mathrm{N}$ and $23^{\circ} \mathrm{N}$, and weaker variability along the east coast between $16^{\circ} \mathrm{N}$ and $19^{\circ} \mathrm{N}$ (Fig. 1.b), are accurately depicted by the model (Fig. 1.f). However, the modeled exhibits a weaker SST variability than the AVHRR in the northern basin with a smaller standard deviation. The mean and standard deviation of model SSH are comparable to those of the daily AVISO gridded SSH product (available at http://www.aviso.altimetry.fr/en/data/data-access/aviso-opendap/opendap-adtproducts.html). The model well reproduced the south-to-north SSH gradient in the basin (Fig. 1.g) compared with the AVISO data (Fig. 1.c). The model SSH variability is slightly weaker than what is observed by AVISO, especially towards the Saudi coast 
in the central and northern Red Sea. The larger variability of modeled SSH in the central and northern basins is likely the signature of a strong eddy variability (Fig. 1.h), which is not always represented by AVISO data (Fig. 1.d). The discrepancy may result from sub-mesoscale features in the model outputs that are not represented by the $0.25^{\circ}$ AVISO data. In addition, the merged gridded AVISO product is generally produced from low-coverage of daily along-track data (Zhan et al. 2016), which may underestimate the eddy intensities (Zhai and Bower 2013).

\section{Ensemble Assimilation Schemes and Implementation}

\subsection{Ensemble Kalman filtering}

Data Assimilation serves to incorporate observational data with numerical models to best estimate the state of the ocean (Edwards et al. 2015). It is mainly used for forecasting purposes, but also for developing ocean reanalysis products, parameter estimation, uncertainty quantification, etc. State-of-the-art ocean data assimilation schemes are now well established following two directions depending on how the data are assimilated into the model. The variational approach seeks for the deterministic model trajectory that best fits all available observations by tuning some uncertain model parameters. The model-data fit is measured by a well-chosen objective function that is optimized based on its gradients calculated using the adjoint of the ocean model (Ledimet and Talagrand 1986). The filtering approach sequentially updates the model forecasts every time new observations are available based on prior errors estimates on the model forecast and assimilated data (Hoteit et al., 2015). The most widely used sequential assimilation schemes are the ensemble Kalman filter (EnKF) and its variants (Edwards et al. 2015; Evensen 2004; Hoteit et al. 2002; Tippett et al. 2003). These are Monte Carlo-based variants of the famous Kalman filter (KF) designed for nonlinear and computationally demanding models (Evensen 1994). In contrast with the variational methods, the Kalman methods are non-intrusive (do not require the development of an adjoint model), and are therefore easier to implement. 
A crucial aspect of any data assimilation scheme is a good description of the forecast error covariance, often referred to as the background covariance, which describes how the model-observation misfits are projected into the state space to correct the forecast. When the system is linear and errors statistics are Gaussian, the KF provides an optimal way to sequentially estimate the time evolution of the forecast state and its background covariance according to the system dynamics (Hoteit et al. 2015). To enable the implementation of the KF for data assimilation into realistic high-dimensional and nonlinear ocean GCMs, Evensen (1994) proposed to represent the forecast statistics (first two moments) by an ensemble of state vectors, called ensemble members. Given an ensemble of model forecasts $\mathbf{X}^{f}=$ $\left[x_{1}^{f}, x_{2}^{f}, \ldots, x_{N}^{f}\right]$, estimates of the forecast state and its background covariance are then taken as the sample ensemble mean and covariance,

$$
\overline{\boldsymbol{x}}^{f}=\frac{1}{N} \sum_{i=1}^{N} \boldsymbol{x}_{i}^{f} \quad \text { and } \quad \mathrm{P}^{f}=\frac{1}{N-1} \mathbf{X}^{\prime}\left(\mathbf{X}^{\prime}\right)^{\mathrm{T}},
$$

where $\mathbf{X}^{\prime}=\left[\boldsymbol{x}_{1}^{f}-\overline{\boldsymbol{x}}^{f}, \boldsymbol{x}_{2}^{f}-\overline{\boldsymbol{x}}^{f}, \ldots, \boldsymbol{x}_{N}^{f}-\overline{\boldsymbol{x}}^{f}\right]$ is the ensemble of anomalies. This provided a particularly efficient framework to estimate the forecast error covariance for adequate weighting of the forecast in the assimilation, to account for various sources of model errors, and to quantify the uncertainties in the estimated solution (Hoteit et al. 2013). This study focuses on the ensemble Kalman methods, which we implement here based on the Data Assimilation Research Testbed (DART) package. Our goal is to develop an efficient, in term of computational cost and performances, assimilation system for reconstructing and forecasting the spacetime circulation of the Red Sea and to quantify the uncertainties in the estimated fields.

As the KF, EnKFs operate as a succession of forecast and analysis steps. In the forecast step, the ensemble members are integrated with the dynamical model to the time of the next available observations. In the analysis step, the forecasted members are adjusted by the incoming observations using the KF update step:

$\boldsymbol{x}^{a}=\boldsymbol{x}^{f}+P^{f} \boldsymbol{H}^{T}\left(\boldsymbol{H} P^{f} \boldsymbol{H}^{T}+R\right)^{-1}\left[\boldsymbol{y}-H\left(\boldsymbol{x}^{f}\right)\right]$, 
where $P^{f}$ is the forecast error covariance, $R$ is the observational error covariance, $y$ is the observational vector, and $\boldsymbol{H}$ is the linearized form of the observational operator $H$ (in our setting, the assimilated SST and SSH data are model variables, so that $H$ is linear).

EnKF methods were classified into stochastic or deterministic techniques, depending on whether the observations were perturbed, or not, before assimilation (Tippett et al. 2003). Deterministic filters, such as the EAKF, became more popular for data assimilation in oceanography (Edwards et al. 2015) and meteorology (Houtekamer and Zhang 2016), to avoid introducing noise from the under-sampling of the observational error covariance with a small ensemble (Altaf et al. 2014; Hoteit et al. 2015; Nerger et al. 2005). The performance of an EnKF greatly depends on the representativeness of its ensemble members, which should adequately describes the statistics of the state estimates errors.

The EAKF update step is based on the following equations (Anderson 2001):

$$
\begin{aligned}
& \overline{\mathrm{x}}^{\mathrm{a}}=\mathrm{P}^{\mathrm{a}}\left[\left(\mathrm{P}^{\mathrm{f}}\right)^{-1} \overline{\mathrm{x}}^{\mathrm{f}}+\mathrm{H}^{\mathrm{T}} \mathrm{R}^{-1} \mathrm{y}^{\mathrm{o}}\right], \\
& \mathrm{P}^{\mathrm{a}}=\left[\left(\mathrm{P}^{\mathrm{f}}\right)^{-1}+\mathrm{H}^{\mathrm{T}} \mathrm{R}^{-1} \mathrm{H}\right]^{-1}, \\
& \mathrm{x}_{\mathrm{i}}^{\mathrm{a}}=\mathrm{A}^{\mathrm{T}}\left(\mathrm{x}_{\mathrm{i}}^{\mathrm{f}}-\overline{\mathrm{x}}^{\mathrm{f}}\right)+\overline{\mathrm{x}}^{\mathrm{a}}, \mathrm{i}=1, \ldots, \mathrm{N},
\end{aligned}
$$

where $x_{i}^{f}$ and $x_{i}^{a}$ are individual members of the forecast and analysis ensemble and $\overline{\mathrm{x}}^{\mathrm{a}}$ and $\overline{\mathrm{x}}^{\mathrm{f}}$ their respective means, and $\mathrm{N}$ is the ensemble size. Equations (i) and (ii) compute the analysis state $\overline{\mathrm{x}}^{\mathrm{a}}$ and its error covariance matrix $\mathrm{P}^{\mathrm{a}}$ from the forecast ensemble mean $\overline{\mathrm{X}}^{\mathrm{f}}$ and covariance $\mathrm{P}^{\mathrm{f}}$, exactly as in the Kalman filter. The analysis members are then generated using Equation (iii) in such a way to exactly match $\overline{\mathrm{x}}^{\mathrm{a}}$ and $\mathrm{P}^{\mathrm{a}}$, which are the constraints for selecting the matrix A.

The EAKF formulation enables updating the ensemble with the model to track changes in the ocean dynamics, which should be particularly useful in regions subject to important spatial and temporal variability. 
EAKFs may suffer from the collapse of their ensembles members (towards the ensemble mean) (Furrer and Bengtsson 2007), especially when the forecast model is not integrated with stochastic perturbations to account, for instance, for uncertainties in the forcing and/or parameters. One simple approach that was proven efficient to mitigate this is to amplify the ensemble spread by multiplying by an inflation factor that is larger than 1 (Anderson 2001; Hamill and Whitaker 2011; Hoteit et al. 2002). Another challenge in ensemble Kalman methods is the spurious correlations in the forecast error covariance that is inherited from the use of small ensembles in practice (Houtekamer and Mitchell 1998). The low-rank nature of the covariances of such ensembles may further extend the impact of an observation to faraway points from its location, which may severely limit the filter ability to fit the data (Hamill et al. 2001). This problem can be generally efficiently addressed through the localization technique (Fu et al. 2011; Hamill et al. 2001; Houtekamer and Mitchell 2001; Oke et al. 2007). The basic idea is to restrict the impact of an observation to nearby points only, or equivalently, trim long-range correlations from the ensemble covariance (Sakov and Bertino 2011). In practice, large inflation and strong localization may, however, respectively weaken the stability of the assimilation system, and introduce undesirable small-scale features into the analysis when the observations are sparse (Hamill and Snyder 2000).

Integrating large ensembles with an ocean GCM is computationally demanding. Following the optimal interpolation (OI) approach in data assimilation, which uses a static pre-selected background covariance in the update step, ensemble OI (EnOI) methods were proposed (Evensen 2003; Hoteit et al. 2002; Oke et al. 2002). EnOI is a very cost effective alternative to an EnKF in which the static background covariance is estimated as the sample covariance matrix of an adequately preselected ensemble, generally representing the error growing modes, or describing the variability of the studied system. This formulation does not suffer from the ensemble collapse problem, but its performance may be limited during periods of strongly changing dynamics that are generally not well described by a static background (Hoteit and Pham 2004; Hoteit et al. 2002). EnOI has a very similar 
algorithm as an EnKF, except that only the analysis state, and not the entire ensemble, is integrated with the model during the forecast step. The method was found to provide good performances compared to an EAKF at fraction of the computing $\operatorname{cost}^{1}$ (Hoteit et al. 2002; Oke et al. 2007; Sakov and Sandery 2015).

A completely static background error covariance may not ideally describe the variable patterns of the ocean flow in different seasons, and periods in between. To represent the flow-dependence in term of seasonality, Xie and Zhu (2010) proposed to use EnOI to assimilate Argo profiles in a hybrid coordinate ocean model (HYCOM) with an ensemble selected at every assimilation cycle from monthly climatology fields with a three-month moving window around the assimilation time. The same scheme was latter adopted by Xie et al. (2011) and Lyu et al. (2014) for assimilating SLA data in the South China Sea. In this study, we implemented a similar EnOI scheme but selecting the ensemble on a monthly basis from a climatological dataset of the Red Sea circulation that is assumed to describe the variability of the system. The Red Sea climatology was simulated from a long-term historical model simulation as described in more details in the next section. We refer to the EnOI with seasonally varying ensemble as the seasonal-EnOI (or SEnOI).

\subsection{Implementation within DART}

EAKF, EnOI and SEnOI were implemented in fully parallel mode (at the forecast and the anslysis steps) using the Data Assimilation Research Testbed (DART). DART is a portable software for ensemble data assimilation developed at the National Center for Atmospheric Research (NCAR) (Anderson et al. 2009). It builds on a series of interface routines that incorporate a forecast model and different types of observations, and can be used with a variety of algorithms to update the ensemble, including for instance the (stochastic) EnKF and the EAKF. DART is configured to integrate and update the ensemble members in parallel, exploiting the serial

\footnotetext{
1 in CPU time and not necessarily in real time as the ensemble members can be integrated in parallel.
} 
formulation of the Kalman filter update step (Anderson and Collins 2007). It is further equipped with advanced inflation/localization techniques that are important to enhance the performance of an ensemble-based data assimilation system. It has been successfully implemented in various atmospheric and oceanic applications (Aksoy et al. 2009; Hoteit et al. 2013; Raeder et al. 2012).

DART has been already implemented with the MITgcm for data assimilation and forecasting the loop current in the Gulf of Mexico (Hoteit et al. 2013). A similar system is implemented in this study with some specific adjustments to the Red Sea. The model state vector is composed of the prognostic ocean variables that are needed to initialize the MITgcm, i.e. salinity, temperature, horizontal velocity, and sea surface height fields. We used the EAKF as described by (Anderson 2001; Anderson 2003), and modified some of its routines to enable for EnOI and SEnOI assimilation. The EAKF steps in DART-MITgcm are summarized in the schematic map Fig. 2. Starting from an analysis step, a given initial ensemble $\mathbf{X}^{f}$ is delivered to DART, the mean and covariance of $\mathbf{X}^{f}$ are updated with the filter, based on which the analysis ensemble $\mathbf{X}^{a}$ is then deterministically generated. This is followed by the forecast step, in which each member of the analysis ensemble $\mathbf{X}^{a}$ is integrated with the MITgcm to obtain the new forecast ensemble, which enables to start a new assimilation cycle.

In this study, the initial ensemble was selected from the outputs of the long-term model simulation between 1992 and 2004. Three-day outputs were saved and assigned into 12 datasets according to the time-period between their dates and the beginning of each month. For each month, a total of 252 model outputs were retained. As the experiments start from January-1-2006, the 252 sampled outputs were collected from early December and late January in different years, and the first 50, 100 or 250 records were assembled as the initial, or static, ensemble when running an experiment with those number of members. 
The EnOI steps in DART-MITgcm are schematized in Fig. 3. The filter starts from a given state estimate and an ensemble of model outputs from which we remove the mean to obtain an ensemble of anomalies $\mathbf{X}^{\prime}$. When the new observations become available, one would read $\mathbf{X}^{\prime}$ and compute the "new" forecast ensemble using $\boldsymbol{X}^{f}=\boldsymbol{x}^{f}+\boldsymbol{X}^{\prime}$, which is then sent to DART to compute the analysis state $\boldsymbol{x}^{a}$ (no resampling of a new ensemble is needed here). The MITgcm is then integrated only once to compute the forecast state $\boldsymbol{x}^{f}$. A new assimilation cycle could then be initiated. In our implementation, $\mathbf{X}^{\prime}$ is the same as the EAKF initial ensemble and is kept invariant in time. SEnOI is implemented based in EnOI, except that, its ensemble of anomalies is monthly updated by selecting its members from a climatological dataset consisting of long-term model outputs centered at the beginning of each month.

\section{Experiments Setup and Assimilation Results}

The assimilation experiments were performed over a one year period starting from January-1-2006. Along-track SSH and gridded AVHRR SST were assimilated every 3 days at midnight. The model data misfits were calculated as if all the data were observed at the assimilation time. Since we are using sequential data assimilation schemes, the SSH/SST data were binned at the middle of the assimilation window. The experiments were conducted with different ensemble sizes, inflation factors, and atmospheric forcing conditions.

The ensemble localization technique is applied to remove eventual spurious longrange correlations that may appear from the use of small ensembles and to increase the rank of the forecast error covariance. A covariance localization cutoff radius of $0.05 \mathrm{rad}$ (about $300 \mathrm{~km}$ depending on the latitude) is chosen from a series of assimilation runs with different localization scales (not shown), providing good and robust assimilation results. To maintain enough ensemble spread and avoid the ensemble collapse the spread of the anomaly forecast ensemble $\mathbf{X}^{\prime}$, i.e. ensemble covariance, was amplified by an inflation factor, before each analysis step. This is simply implemented by using the following inflated members in the analysis step 
$\boldsymbol{x}_{n}^{f_{-} i n f}=\alpha\left(\boldsymbol{x}_{n}^{f}-\overline{\boldsymbol{x}}\right)+\overline{\boldsymbol{x}} ; n=1, \ldots, N$

where $\alpha$ is an inflation factor generally chosen to be slightly greater than 1 .

The choice of the ensemble from which the forecast error covariance $\mathrm{P}^{\mathrm{f}}$ is estimated is key for designing an efficient sequential ensemble assimilation system. In all the experiments presented hereafter, the initial ensemble of the EAKF is selected from a set of January climatological fields, i.e., the members are selected from the January outputs of a long-term model run. In the EnOI, this ensemble is kept invariant in time while in SEnOI the ensemble is reselected from the model outputs for the corresponding month.

\subsection{EAKF Results}

Sensitivity to ensemble size

The objective of an EAKF scheme is to minimize the variance of the analysis error, which is expected to decrease as the ensemble size increases. The choice of the ensemble size is critical to the success of an EAKF assimilation system, and one should balance between ensemble size and computational cost. The ensemble should be large enough to well describe the statistics (mean and spread) of the prior distribution, and to provide a smooth enough covariance between the model state and the observations and avoid severe localization (Mitchell et al. 2002). At the same time, the ensemble size should be reasonable to avoid excessive computing cost. Many studies suggested that, with appropriate localization and inflation, the decrease in the analysis error may stagnate with very large ensembles, suggesting that good performances may be obtained with relatively reasonable size ensembles (Reichle et al. 2002).

To investigate the sensitivity of the EAKF-assimilation system in the Red Sea to the ensemble size, three experiments with 50, 100, 250 ensemble members were performed. An inflation factor of 1.1 and localization radius of about $300 \mathrm{~km}$ were considered in all three experiments. The time-evolution of the root-mean-square errors (RMSEs) between SSH/SST observations and filter forecast/analysis states 
are plotted in Fig. 4. It is clear that for both SSH and SST, the RMSE decreases with larger ensembles. The experiment using 250 members leads to the smallest RMSE for both forecast and analysis, although the RMSE resulting from 50 members is quite reasonable, with an average forecast and analysis RMSEs of $0.71{ }^{\circ} \mathrm{C} / 0.08 \mathrm{~m}$ and $0.64{ }^{\circ} \mathrm{C} / 0.07 \mathrm{~m}$ for SST and $\mathrm{SSH}$, respectively. Nevertheless, the improvements resulting from increasing the ensemble size from 100 to 250 are generally not very significant (especially for SST), and this is also reflected in their ensemble spreads. The SST and SSH ensemble spreads stabilize after the first 30 assimilation cycles, (about three months) reaching minimal values of about $0.1^{\circ} \mathrm{C}$ and $0.01 \mathrm{~m}$ in the winter season, before they slightly increase during the summer season. The SST and SSH analysis RMSEs are about $0.1^{\circ} \mathrm{C}$ and $0.01 \mathrm{~m}$ lower than the corresponding forecast RMSEs, respectively, suggesting that the data are properly assimilated into the model. The RMSE of SSH analysis is lower than that of the AVISO gridded data, which has been generated by merging different satellite missions' measurements (Ducet et al. 2000).

Increasing the ensemble size may increase the risk of collapse of the ensemble assimilation system; this is exactly what happened after 61 assimilation cycles (or six months) for the run with 250 members, when the MITgcm was not able to complete the integration of one of the ensemble members and diverged. In each assimilation cycle, the analysis ensemble increment, which is introduced by the Kalman gain matrix, may introduce some dynamically unstable realizations that are not compatible with the model physics (Gottwald 2014). This imbalance can be further more severe with localization and inflation (Oke et al. 2007). Improving the dynamical consistency of the ensemble members and developing efficient online schemes to replace unstable members are two of the directions we are planning to explore in order to enhance the robustness of the system. Hereafter, we will limit the ensemble size to 100 as this seems to provide enough representative ensembles in order to obtain good and robust ensemble assimilation performances.

Sensitivity to inflation 
The value of the inflation factor may depend on the dynamics of the model and the studied region, and on the configuration of the assimilation system (including the ensemble size and filter (Hamill et al. 2001). Here we conduct trial and errors experiments to set the value of the inflation factor. Sophisticated adaptive inflation schemes were suggested for online space-time tuning of the value of inflation (Altaf et al. 2014; Anderson et al. 2009; Hoteit et al. 2002), but these also require to be configured to the studied region and were not considered in this study.

To investigate the sensitivity of the MITgcm-EAKF Red Sea assimilation system to the inflation factor, four ensemble assimilation experiments with different values of inflation factors, 1.0 (no inflation), 1.05, 1.1 and 1.2, were conducted using the same ensemble size of 100 members. The time-evolution of SST and SSH RMSEs for the forecast and analysis fields, and the corresponding forecast ensemble spreads are plotted in Fig. 5. The results suggest that the overall performance of the MITgcmEAKF assimilation system in the Red Sea is quite dependent on the choice of the inflation factor. A remarkable improvement in the filter performance in term of RMSE is achieved using inflation, as compared to the filter results without inflation (inflation $=1.0$ ). The accuracy of the filter estimates particularly improves with inflation in summer for both SST and SSH. However, increasing the inflation factor from 1.1 to 1.2 is not very beneficial, or even occasionally contributing negatively, to the system behavior. The ensemble spread of SSH and SST (Fig. 5.e and Fig. 5.f) decreases over time, with the largest decreases during the first few assimilation cycles, before stabilizing in later cycles. The ensemble spreads are then maintained at levels of about $0.15^{\circ} \mathrm{C}$ and $0.01 \mathrm{~m}$ for SST and SSH, respectively. With inflation, the ensemble spread decreases at a slower pace, but tends to diverge after some cycles, depending on the value of the used inflation factor. It is necessary to note that a larger inflation factor noticeably reduced the analysis RMSEs, but not always the forecast RMSEs, particularly for SSH. This probably implies that an EAKF system with larger inflation factor may overfit the observational data, providing analysis fields featuring some dynamically unbalanced features. 
Although inflation generally helps maintaining the ensemble spread, large inflation factors may deteriorate the system behavior and even cause divergence. The experiment using an inflation factor of 1.2 stops after 55 assimilation cycles only. The large anomaly imposed by a large inflation factor may cause runaway increase in some states trajectories compared to those purely integrated with the model. This may force the analysis state to overfit the observations in regions where data are available, leading to strong contrast with regions that are not covered by observations (Luo and Hoteit 2013). The unphysical and imbalanced inflated variances may lead to large signal-to-noise ratios in the fits, which could impose spurious adjustments to the ensemble and further lead to poor forecasts (Hoteit et al. 2013).

\subsection{Sensitivity to the choice of the background ensemble}

\section{EAKF vs. EnOI vs. SEnOI}

To investigate the behavior of the Red Sea ensemble assimilation system with different choices of ensemble schemes, assimilation experiments were conducted using the EAKF, EnOI and SEnOI. Following the results of Section 4.1, the results of the EAKF with 100 ensemble members are used as a reference to evaluate the performances of the two other ensemble schemes.

In contrast with the EAKF, which requires integrating all ensemble members with the MITgcm in the forecast step, EnOI and SEnOI only run the model once, to compute the forecast state starting from the filter analysis, regardless of the ensemble size. One can therefore implement the EnOI schemes with large ensemble members without significant increase in the computational cost. Here we compared the performances of EnOI and SEnOI with 250 ensemble members with those of EAKF with 100 members.

The results are shown in Fig. 6. In term of spread, both EnOI schemes exhibit much larger ensemble spreads, whether calculated from a static or a seasonally varying ensemble, as compared to that of EAKF (Fig. 6.e and Fig. 6.f). The larger spreads of 
the forecast ensembles of the EnOI schemes suggest, in some sense, larger forecast errors, which pushes the filter's analysis more towards the observations. This is reflected in the analysis RMSE for both SST (Fig. 6.c), and SSH (Fig. 6.d), where the EAKF clearly exhibits a larger RMSE than those of the EnOIs. The differences between the EnOI and SEnOI in terms of their SST and SSH analysis RMSEs are surprisingly not significant, with the latter being comparable to that of the gridded AVISO product.

In term of forecast, the performances of the three ensemble schemes are comparable for SST. This is not surprising because the Red Sea SST is dominated by the atmospheric forcing and boundary conditions, which are identical in all three experiments. In forecasting SSH, however, EAKF significantly outperforms both EnOI and SEnOI, which further exhibit an erratic behavior despite using a smaller ensemble. SEnOI generally provides better forecasts than EnOI, except during the winter season, where the static ensemble seems to be well representative of this period.

Remote sensing SSH is one of the most used data to describe mesoscale eddies activities in the ocean, and, in practice, provides the most compelling measurements to constrain modeled eddies. The repeat-cycle of satellite altimeters over the Red Sea ranges between 10 to 35 days, which is much longer than the 3-day assimilation window considered in this study. Therefore, unlike the SST observations that are always mapped on the same regular grids, the number and locations of along-track SSH observations vary with the satellites tracks at each analysis step. This means that the forecast SSH RMSE is often evaluated against observations that are not located in the regions where the previous observations were assimilated to produce the most recent analysis (based on which the forecast was computed). One could then consider the SSH altimeter data as independent data to evaluate the filters forecasts. The much better EAKF forecasts suggest better ability to reproduce the hydrodynamics of the Red Sea with a flow-dependent ensemble. In the EnOI and SEnOI experiments, the forecast SSH RMSE (Fig. 6.b) is quite larger than that of the 
analysis SSH (Fig. 6.d), indicating that the ocean model didn't adjust fast enough to the (usually large) increments imposed by the no-flow-dependent backgrounds of the EnOI schemes, which probably caused some dynamical imbalances with the ambient water.

We also analyze the forecasts SSH as they result from EnOI and SEnOI. Eddies activity is the most dominant and energetic component of the Red Sea variability, which is usually distinguished from the mean flow as perturbations and can be characterized from the fields of anomalies (Zhan et al. 2016). This happens to be similar to the generation process of the forecast error covariance $\left(\mathrm{P}^{f}=\frac{1}{N-1} \mathbf{X}^{\prime}\left(\mathbf{X}^{\prime}\right)^{\mathrm{T}}\right)$ using an ensemble of anomalies, whose members are selected from climatological fields composed of 15-year model outputs. Therefore, the 250 selected ensemble members should be able, to some extent, to represent the eddy variability in the Red Sea. In this case, the constructed $\mathrm{P}^{f}$ would possibly describe eddy features that happen to be not observed by the sparse altimeter data, or not captured by the forecast state. This could possibly explain why the EnOI with a static ensemble selected in January climatology was able to provide reasonable results. Furthermore, as the Red Sea eddies exhibit a seasonal variability (Zhan et al. 2014; Zhan et al. 2016), the SEnOI generally out-performs EnOI, particularly in summer, leading to a smaller SSH forecast RMSE. However, the overturning circulation and subsurface intrusion water from the Gulf of Aden also vary with seasons, and these features cannot be well evaluated based on SST and SSH data. A more robust evaluation would be to also assess the results of the different ensemble assimilation schemes against in-situ profiles in different seasons, but this requires much longer assimilation runs over several years.

As an example, the spatial distributions of the forecast and analysis states on June-62006 as estimated by the three ensemble assimilation schemes are compared with remote sensing observations of SSH (Fig. 7) and SST/temperature profile (Fig. 8). The SSH and SST observations are extracted from gridded AVISO and the AVHRR products, respectively. Forecasts from all three schemes agreed well with the 
remote sensing data, and additionally provided more detailed mesoscale and submesoscale features in the basin than the gridded products. In particular, compared with EAKF, the EnOI schemes introduced stronger eddy activities in the northern Red Sea. This can be clearly seen from the dark blue patches of SSH (Fig. 7.a-c), the filament features in SST (Fig. 8.a-c) and the corresponding doming of temperature profile (Fig. 8.h-j). EnOI and SEnOI also introduced stronger eddies around the altimetry data tracks than EAKF with more pronounced SSH increments (Fig. 6.h-j). This resulted from the larger ensemble spread, which assigned more weight to the observations in the EnOI schemes (Fig. 6.e-f). Therefore, EnOIs were more likely to fit the observations and to introduce new features in the analysis fields. These used static ensembles of anomalies that maintain the variability of the Red Sea state (mainly featured with eddy signal) throughout the simulation, while in EAKF, the ensemble tends to converge towards the mean despite the use of inflation, leading to updates that are less impacted by the observations, on basin scales.

Fig. 9 shows the spatial distribution of the ensemble spread on June-6-2006 in the three schemes. The EAKF spread is significantly smaller than the others. The EnOI ensemble is selected from January climatology, while the SEnOI ensemble in this example is updated from June climatological fields. We noticed that the ensemble spread of SSH in SEnOI exhibits stronger eddy variability than that in EnOI, but the ensemble spread of temperature in EnOI (Fig. 9.e and Fig. 9.h) is larger than that of SEnOI (Fig. 9.f and Fig. 9.i), both on the surface and in the upper layers, the latter of which is probably related to a deeper mix layers in winter. The larger SST spread is explained by the stronger interannual atmospheric variability in the winter and the sensitivity of SST to the atmospheric conditions.

\subsection{Dynamical Balance}

It is important to investigate the dynamical balance of the state estimates of the ensemble assimilation schemes. In the analysis fields, the flow is expected to satisfy the geostrophic balance for large-scale and mesoscale phenomenon. In other words, 
the zonal and meridional residue terms $\alpha_{x}$ and $\alpha_{y}$ (Reynolds stress divergences, advection and acceleration terms) defined in the following momentum equations are expected to be small:

$$
\alpha_{x}=\frac{\partial \emptyset}{\partial x}+\mathbb{u} \cdot \nabla u-f v, \alpha_{y}=\frac{\partial \emptyset}{\partial y}+\mathbb{u n} \cdot \nabla \mathrm{v}+f u,
$$

where $\emptyset, \llbracket, u, v, f$ represent the dynamic pressure, the 3-D velocity, the zonal and meridional velocity and the Coriolis parameter, respectively. The residue terms are calculated from the analysis fields, which were updated based on available observations during the assimilation. The zonal and meridional residue terms at a $50 \mathrm{~m}$ depth and the relative comparison between the Coriolis term and the horizontal pressure gradient term are plotted in Fig. 10.

In all these assimilation runs, the geostrophic balance of the analysis fields is well satisfied and the imbalance accounts only for a small portion of the total term. In particular, the new introduced eddies in the EnOI and SEnOI at 20-22 ${ }^{\circ} \mathrm{N}$ (Fig. 7.h-j) are dynamically balanced. The increments derived in these ensemble-based data assimilation systems can be expressed as $\boldsymbol{x}^{a}-\boldsymbol{x}^{f}=P^{f} \boldsymbol{H}^{T}\left(\boldsymbol{H} P^{f} \boldsymbol{H}^{T}+R\right)^{-1}[\boldsymbol{y}-$ $\left.H\left(\boldsymbol{x}^{f}\right)\right]=\mathbf{X}^{\prime} \boldsymbol{c}$, given the forecast error covariance $\mathrm{P}^{f}=\frac{1}{N-1} \mathbf{X}^{\prime}\left(\mathbf{X}^{\prime}\right)^{\mathrm{T}}$ and an $N$ dimensional column vector $\boldsymbol{c}$. The increment at any analysis step is therefore essentially a linear combination of $\mathbf{X}^{\prime}$. The long-term simulation outputs, from which the ensemble members are sampled, are geotropically equilibrated and adjusted with the model dynamics. The same also should hold for the members of the ensemble of anomalies $\mathbf{X}^{\prime}$. Therefore, the increment naturally satisfies the geostrophic balance, which can be seen in Fig. 7.h-j, where the velocity increments correspond to the SSH increments. Although the large- and meso-scale balances are warranted, the filter estimates may exhibit some imbalance in small-scale dynamics due to, for instance, stress divergences, acceleration. Localization and inflation may also distort the balance. This inevitable imbalance seems to be generally quite weak and the model is generally able to dynamically adjust it. However, the weak imbalance may be amplified by the magnitude of the increment $c$, which eventually 
imposes pronounced changes to the analysis fields. If the imbalance is large to top the robustness of the model, the model may sometimes blow up when integrating the members during the forecast step and the assimilation system would break down. In addition, the time-evolution of the basin averaged total residue $(\alpha=$ $\sqrt{\alpha_{x}^{2}+\alpha_{y}^{2}}$, not shown) suggests that the EAKF estimates are more dynamically balanced than the OI solutions, often imposing less increments on the forecast because of its smaller ensemble spread.

\subsection{Sensitivity to the atmospheric forcing: NCEP vs. ECMWF}

To test the sensitivity of the assimilation system to the atmospheric forcing product, we compared the results of two assimilation experiments that have been conducted with the EAKF under identical conditions and forced with ECMWF and NCEP fields. The experiments are initialized with an ensemble size of 100 members selected from January climatological fields. The performances of the two experiments are quite comparable in terms of SST and SSH RMSEs (Fig. 11.a-d). To investigate whether the model without assimilation is sensitive to the atmospheric forcing, we carried out two free model runs respectively forced with ECMWF and NCEP. The free-run results shown in Fig. 11.e and Fig. 11.f suggest that the model is sensitive to the atmospheric conditions, and that NCEP and ECMWF do force different circulations in the Red Sea. The disagreement is most pronounced in the SST, probably implying considerable differences in the ECMWF and NCEP heat flux fields in this region. Clearly, data assimilation reduces the SST forecast RMSE (Fig. 11.a and Fig. 11.e) in both experiments, with the $1.1^{\circ} \mathrm{C}$ and $1.2^{\circ} \mathrm{C}$ RMSE of the free run forced with ECMWF and NCEP respectively reduced to $0.7{ }^{\circ} \mathrm{C}$ and $0.6{ }^{\circ} \mathrm{C}$ in the assimilation experiments. Even though the different atmospheric conditions may force different circulation patterns in the Red Sea, the assimilation of remotely sensed SSH and SST data is capable to control the system, at least in the upper layer, and to adjust its circulation according to the available observations. 
As atmospheric conditions have a major impact on SST, we further investigate the spatial distribution of the temperature field under different forcing conditions, in the assimilation and free-run experiments. As depicted in Fig. 12 for two examples in August and in December 2006, the EAKF estimates clearly features stronger vertical variability than the free-run outputs. This is most likely related to the eddies that have been introduced by the filter. In particular, the EAKF forced with both ECMWF and NCEP exhibit some doming of isothermal in the central Red Sea in August (Fig. 12.e,f) and in the northern basin in December (Fig.12.m,n), which are much weaker in the free run forced with ECMWF (Fig. 12.g,o) and hardly seen in the free run forced with NCEP (Fig. 12.h,p). In addition, in the two EAKF assimilation experiments forced with different atmospheric conditions, although little difference is found in their SST RMSEs, and their dominant vertical structures are quite comparable, some of their vertical features are still different. For instance, the slightly depressing isothermal of the southern Red Sea in the ECMWF run (Fig. 12.e) differs from the doming isothermal in the NCEP run (Fig. 12.f) in August, suggesting that atmospheric conditions may still influence deep-water structures in an EAKF system, even when the upper layer is well conditioned by the assimilated data.

\section{Summary and Discussion}

This study presented the development of an ensemble data assimilation system for the Red Sea, and investigated its sensitivity to the choice of the ensemble, and to filtering parameters and atmospheric forcing. The system is composed of the MITgcm configured at eddy-resolving resolution, and the DART ensemble software to assimilate available observations. Along-track RADS SSH data and gridded AVHRR SST product were assimilated using a three-day window. We investigated the performances of an ensemble Kalman filter, the EAKF as implemented in DART in fully parallel mode, and based on which we have implemented a variant with static covariance, EnOI scheme. The latter does not integrate the ensemble with the MITgcm, offering drastic reduction in computational cost. To deal with the dominant seasonal variability of the Red Sea circulation, the EnOI ensemble was monthly 
updated by selecting new members from a given climatology of long-term model outputs. This scheme was referred to as seasonal-EnOI, or SEnOI.

An ensemble of 100 members was found enough to obtain good forecasting skills with the EAKF at reasonable computational cost. Increasing the ensemble size to 250 did not improve much the EAKF performances. Inflation is used to artificially increase the ensemble spread and to account for various sources of uncertainties that are either not accounted for or not optimally prescribed in an assimilation system, such as modeling uncertainties, inputs and forcing, filtering approximations, etc. (Hoteit et al. 2002). As such, the value of the inflation factor is system dependent and may vary from one application to another. In our case, we found that an inflation of 1.1 provides the best results, which may give some indications about a suitable value of inflation factor to try in a similar setting. Note that, when the error cross-correlations are well described by the ensemble, the RMSE generally decreases with increased inflation, but of course up to a certain threshold after which one may encounter observations over-fitting issues with more inflation (i.e. larger ensemble spread). A too large inflation might in this case trigger dynamical inconsistency issues, often causing the ensemble to collapse, as we also see in our experiments. The assimilation system was also found not very sensitive, at least for the assimilated surface layer and the dominant vertical structures, to the atmospheric forcing, NCEP or ECMWF fields. Conditioned on the available remote sensing observations, the system is able to adjust the initial state to provide equivalent forecasting products regardless of the forcing product.

Assimilation results from experiments focusing on the relevance of updating the ensemble with the ocean model (EAKF) suggest that, with adequate choice of the static ensemble, (seasonal) EnOI can provide comparable, and even sometimes superior (especially for SST), analysis results. It is important to point out that despite the larger analysis SST RMSE in EAKF (Fig. 6 (c)), its forecast SST RMSE (Fig. 6 (a)) is generally better than both EnOI schemes, suggesting proper assimilation of 
the SST by the EAKF. After all, the best measure of an assimilation system performance is the forecast error, and not the analysis error. The performance of the EAKF with SST could be expected as our analysis suggests that the EAKF ensemble was shown not to carry enough spread for SST, as compared to the other two EnOI schemes (Fig. 6 (e)). In our experiments, increasing inflation improved the EAKF results, but also often caused the collapse of some of the ensemble members, and thus the filter. A better treatment of the system uncertainties, e.g. through the use of forcing perturbations, is likely to enhance the EAKF performance for SST.

In addition, EAKF clearly provide better forecasts for SSH. A noticeable feature of EnOI and SEnOI, that was seen from the difference between the SSH forecast and analysis RMSEs, is their capability of imposing strong eddies signatures on their analysis fields, but these dynamics seem not quite in line with the ambient water and were often quickly dissipated. Although the geostrophic balance is generally expected in the filters analyses, this may hurt the model forecasting skill when the filter adjustment causes fast-propagating inertial and internal gravity waves. In such an ensemble data assimilation system, the selection of the ensemble subspace is key. It defines the space onto which the model updates are projected. In the Red Sea, the spatial scales of the most energetic and variable components of ocean dynamics (e.g. eddies) are mostly composed of mesoscales or sub-mesoscales (10 km or less) features, which can be described by the anomaly of the mean flow. Such information is reflected from the definition of the forecast error covariance in an ensemble assimilation system. This is a convincing theoretical basis for applying the (seasonal) EnOI method with a static ensemble of state anomalies to enforce eddy variability in the analysis. In contrast, the data-conditioned and flow-dependent ensemble in the EAKF may sometimes lack information about eddies, which may limit its ability to reintroduce them in the analysis.

In the ocean, eddies are often greatly under-sampled due to the sparse available observations, and are therefore likely to be missed given their relatively short spatial scales. In an assimilation system, when eddies happen to be poorly 
represented in the forecast ensemble, the analysis step is expected to somehow reintroduce these features, provided enough observations coverage. Since the increment between the analysis and the forecast is essentially represented by a linear combination of ensemble members, one should select an ensemble that well represent the eddies, incorporating flow-dependent information to track changes in the system dynamics, and that does not collapse over time. A mixture of dynamically evolving and static ensemble, based on the so-called Hybrid ensemble schemes, may entertain both features and will be one of our targets for the future development of the system.

Another limitation of the EAKF is that the assimilation run often terminates when one, or more, members diverge during the integration with the dynamical model, probably caused by some imbalances introduced in the analysis step. To enhance the robustness of the EAKF against divergence, we will further develop the system to enable for automatic replacement of diverged members by new members to be selected from a given "dictionary" of system state realizations. This will require introducing an optimal selection strategy that is suitable to the unique nature of the circulation and eddy activities in the Red Sea. Such a strategy would definitely benefit from the availability of independent adequate set of observations (e.g. drifters, HF radar, glider data, etc.), which is presently being deployed in the Red Sea. 


\section{Acknowledgment}

This research work was supported by King Abdullah University of Science and Technology (KAUST), Saudi Arabia and the Saudi ARAMCO Marine Environmental Research Center at KAUST (SAMERCK). The research made use of the resources of the Super computing Laboratory and computer clusters at KAUST. 


\section{References}

Aksoy A, Dowell DC, Snyder C (2009) A Multicase Comparative Assessment of the Ensemble Kalman Filter for Assimilation of Radar Observations. Part I: Storm-Scale Analyses Mon Weather Rev 137:1805-1824 doi:10.1175/2008mwr2691.1

Altaf MU, Butler T, Mayo T, Luo X, Dawson C, Heemink AW, Hoteit I (2014) A Comparison of Ensemble Kalman Filters for Storm Surge Assimilation Mon Weather Rev 142:2899-2914 doi:10.1175/Mwr-D-13-00266.1

Anderson J, Hoar T, Raeder K, Liu H, Collins N, Torn R, Avellano A (2009) THE DATA ASSIMILATION RESEARCH TESTBED A Community Facility B Am Meteorol Soc 90:1283-1296 doi:10.1175/2009bams2618.1

Anderson JL (2001) An ensemble adjustment Kalman filter for data assimilation Mon Weather Rev 129:2884-2903

Anderson JL (2003) A local least squares framework for ensemble filtering Mon Weather Rev 131:634-642 doi:Doi 10.1175/15200493(2003)131<0634:Allsff>2.0.Co;2

Anderson JL, Collins N (2007) Scalable implementations of ensemble filter algorithms for data assimilation J Atmos Ocean Tech 24:1452-1463 doi:10.1175/Jtech2049.1

Backeberg BC, Counillon F, Johannessen JA, Pujol MI (2014) Assimilating along-track SLA data using the EnOI in an eddy resolving model of the Agulhas system Ocean Dynamics 64:1121-1136 doi:10.1007/s10236-014-0717-6

Chen CS et al. (2014) Process modeling studies of physical mechanisms of the formation of an anticyclonic eddy in the central Red Sea J Geophys ResOceans 119:1445-1464

Cipollini P et al. (2010) The role of altimetry in coastal observing systems Proceedings of OceanObs 9:181-191

Clifford M, Horton C, Schmitz J, Kantha LH (1997) An oceanographic nowcast/forecast system for the Red Sea J Geophys Res-Oceans 102:2510125122

Ducet N, Le Traon PY, Reverdin G (2000) Global high-resolution mapping of ocean circulation from TOPEX/Poseidon and ERS-1 and-2 J Geophys Res-Oceans 105:19477-19498 doi:Doi 10.1029/2000jc900063

Edwards CA, Moore AM, Hoteit I, Cornuelle BD (2015) Regional Ocean Data Assimilation Annu Rev Mar Sci 7:21-42 doi:10.1146/annurev-marine010814-015821

Eshel G, Naik NH (1997) Climatological coastal jet collision, intermediate water formation, and the general circulation of the Red Sea J Phys Oceanogr 27:1233-1257

Evensen G (1994) Sequential Data Assimilation with a Nonlinear Quasi-Geostrophic Model Using Monte-Carlo Methods to Forecast Error Statistics J Geophys ResOceans 99:10143-10162 doi:Doi 10.1029/94jc00572

Evensen G (2003) The Ensemble Kalman Filter: theoretical formulation and practical implementation Ocean Dynamics 53:343-367 doi:10.1007/s10236003-0036-9 
Evensen G (2004) Sampling strategies and square root analysis schemes for the EnKF Ocean Dynamics 54:539-560 doi:10.1007/s10236-004-0099-2

Fu WW, She J, Zhuang SY (2011) Application of an Ensemble Optimal Interpolation in a North/Baltic Sea model: Assimilating temperature and salinity profiles Ocean Model 40:227-245

Furrer R, Bengtsson T (2007) Estimation of high-dimensional prior and posterior covariance matrices in Kalman filter variants J Multivariate Anal 98:227-255 doi:10.1016/j.jmva.2006.08.003

Gottwald GA (2014) Controlling balance in an ensemble Kalman filter Nonlinear Proc Geoph 21:417-426 doi:10.5194/npg-21-417-2014

Hamill TM, Snyder C (2000) A hybrid ensemble Kalman filter-3D variational analysis scheme Mon Weather Rev 128:2905-2919 doi:Doi 10.1175/15200493(2000)128<2905:Ahekfv>2.0.Co;2

Hamill TM, Whitaker JS (2011) What Constrains Spread Growth in Forecasts Initialized from Ensemble Kalman Filters? Mon Weather Rev 139:117-131 doi:10.1175/2010mwr3246.1

Hamill TM, Whitaker JS, Snyder C (2001) Distance-dependent filtering of background error covariance estimates in an ensemble Kalman filter Mon Weather Rev 129:2776-2790 doi:Doi 10.1175/15200493(2001)129<2776:Ddfobe>2.0.Co;2

Hoteit I et al. (2013) A MITgcm/DART ensemble analysis and prediction system with application to the Gulf of Mexico Dynam Atmos Oceans 63:1-23 doi:10.1016/j.dynatmoce.2013.03.002

Hoteit I, Pham DT (2004) An adaptively reduced-order extended Kalman filter for data assimilation in the tropical Pacific J Marine Syst 45:173-188

Hoteit I, Pham DT, Blum J (2002) A simplified reduced order Kalman filtering and application to altimetric data assimilation in Tropical Pacific J Marine Syst 36:101-127 doi:Pii S0924-7963(02)00129-X

Doi 10.1016/S0924-7963(02)00129-X

Hoteit I, Pham DT, Gharamti ME, Luo X (2015) Mitigating Observation Perturbation Sampling Errors in the Stochastic EnKF Mon Weather Rev 143:2918-2936 doi:10.1175/Mwr-D-14-00088.1

Houtekamer PL, Mitchell HL (1998) Data assimilation using an ensemble Kalman filter technique Mon Weather Rev 126:796-811 doi:Doi 10.1175/15200493(1998)126<0796:Dauaek>2.0.Co;2

Houtekamer PL, Mitchell HL (2001) A sequential ensemble Kalman filter for atmospheric data assimilation Mon Weather Rev 129:123-137 doi:Doi 10.1175/1520-0493(2001)129<0123:Asekff>2.0.Co;2

Houtekamer PL, Zhang F (2016) Review of the Ensemble Kalman Filter for Atmospheric Data Assimilation Mon Weather Rev 0:null doi:doi:10.1175/MWR-D-15-0440.1

Johns WE, Sofianos SS (2012) Atmospherically Forced Exchange through the Bab el Mandeb Strait J Phys Oceanogr 42:1143-1157

Kohl A, Stammer D (2008) Variability of the meridional overturning in the North Atlantic from the 50-year GECCO state estimation J Phys Oceanogr 38:19131930 
Le Henaff M, Roblou L, Bouffard J (2011) Characterizing the Navidad current interannual variability using coastal altimetry Ocean Dynamics 61:425-437

Ledimet FX, Talagrand O (1986) Variational Algorithms for Analysis and Assimilation of Meteorological Observations - Theoretical Aspects Tellus A 38:97-110

Luo XD, Hoteit I (2013) Covariance Inflation in the Ensemble Kalman Filter: A Residual Nudging Perspective and Some Implications Mon Weather Rev 141:3360-3368 doi:10.1175/Mwr-D-13-00067.1

Lyu GK, Wang H, Zhu J, Wang DK, Xie JP, Liu GM (2014) Assimilating the along-track sea level anomaly into the regional ocean modeling system using the ensemble optimal interpolation Acta Oceanol Sin 33:72-82 doi:10.1007/s13131-014-0469-7

Madsen KS, Hoyer JL, Fu WW, Donlon C (2015) Blending of satellite and tide gauge sea level observations and its assimilation in a storm surge model of the North Sea and Baltic Sea J Geophys Res-Oceans 120:6405-6418 doi:10.1002/2015jc011070

Mitchell HL, Houtekamer PL, Pellerin G (2002) Ensemble size, balance, and modelerror representation in an ensemble Kalman filter Mon Weather Rev 130:2791-2808 doi:Doi 10.1175/15200493(2002)130<2791:Esbame>2.0.Co;2

Murray SP, Johns W (1997) Direct observations of seasonal exchange through the Bab el Mandab Strait Geophys Res Lett 24:2557-2560

Nerger L, Hiller W, Schroter J (2005) PDAF - The parallel data assimilation framework: Experiences with Kalman filtering Use of High Performance Computing in Meteorology:63-83 doi:Doi 10.1142/9789812701831_0006

Oke PR, Allen JS, Miller RN, Egbert GD, Kosro PM (2002) Assimilation of surface velocity data into a primitive equation coastal ocean model J Geophys ResOceans 107 doi:Artn 3122

10.1029/2000jc000511

Oke PR, Sakov P, Corney SP (2007) Impacts of localisation in the EnKF and EnOI: experiments with a small model Ocean Dynamics 57:32-45 doi:10.1007/s10236-006-0088-8

Raeder K, Anderson JL, Collins N, Hoar TJ, Kay JE, Lauritzen PH, Pincus R (2012) DART/CAM: An Ensemble Data Assimilation System for CESM Atmospheric Models J Climate 25:6304-6317 doi:10.1175/Jcli-D-11-00395.1

Reichle RH, McLaughlin DB, Entekhabi D (2002) Hydrologic data assimilation with the ensemble Kalman filter Mon Weather Rev 130:103-114 doi:Doi 10.1175/1520-0493(2002)130<0103:Hdawte>2.0.Co;2

Reynolds RW, Smith TM, Liu C, Chelton DB, Casey KS, Schlax MG (2007) Daily highresolution-blended analyses for sea surface temperature J Climate 20:54735496 doi:10.1175/2007jcli1824.1

Sakov P, Bertino L (2011) Relation between two common localisation methods for the EnKF Computat Geosci 15:225-237

Sakov P, Sandery PA (2015) Comparison of EnOI and EnKF regional ocean reanalysis systems Ocean Model 89:45-60 doi:10.1016/j.ocemod.2015.02.003 
Scharroo R, Leuliette EW, Lillibridge JL, Byrne D, Naeije MC, Mitchum GT RADS: Consistent multi-mission products. In, 20132013.

Siddall M, Smeed DA, Matthiesen S, Rohling EJ (2002) Modelling the seasonal cycle of the exchange flow in Bab El Mandab (Red Sea) Deep-Sea Res Pt I 49:15511569

Sofianos SS, Johns WE (2002) An Oceanic General Circulation Model (OGCM) investigation of the Red Sea circulation, 1. Exchange between the Red Sea and the Indian Ocean J Geophys Res-Oceans 107

Sofianos SS, Johns WE (2003) An Oceanic General Circulation Model (OGCM) investigation of the Red Sea circulation: 2 . Three-dimensional circulation in the Red Sea J Geophys Res-Oceans 108

Tippett MK, Anderson JL, Bishop CH, Hamill TM, Whitaker JS (2003) Ensemble square root filters Mon Weather Rev 131:1485-1490

Tragou E, Garrett C (1997) The shallow thermohaline circulation of the Red Sea Deep-Sea Res Pt I 44:1355-1376 doi:Doi 10.1016/S0967-0637(97)00026-5

Triantafyllou G, Yao F, Petihakis G, Tsiaras KP, Raitsos DE, Hoteit I (2014) Exploring the Red Sea seasonal ecosystem functioning using a three-dimensional biophysical model Journal of Geophysical Research: Oceans 119:1791-1811 doi:10.1002/2013JC009641

Tseng KH, Shum CK, Yi YC, Emery WJ, Kuo CY, Lee H, Wang HH (2014) The Improved Retrieval of Coastal Sea Surface Heights by Retracking Modified Radar Altimetry Waveforms Ieee T Geosci Remote 52:991-1001

Vignudelli S, Kostianoy AG, Cipollini P, Benveniste J (2011) Coastal altimetry. Springer Science \& Business Media,

Xie J, Counillon F, Zhu J, Bertino L (2011) An eddy resolving tidal-driven model of the South China Sea assimilating along-track SLA data using the EnOI Ocean Sci 7:609-627 doi:10.5194/os-7-609-2011

Xie JP, Zhu J (2010) Ensemble optimal interpolation schemes for assimilating Argo profiles into a hybrid coordinate ocean model Ocean Model 33:283-298 doi:10.1016/j.ocemod.2010.03.002

Yang L, Lin MS, Liu QH, Pan DL (2012) A coastal altimetry retracking strategy based on waveform classification and sub-waveform extraction Int J Remote Sens 33:7806-7819

Yao FC, Hoteit I, Pratt LJ, Bower AS, Kohl A, Gopalakrishnan G, Rivas D (2014a) Seasonal overturning circulation in the Red Sea: 2 . Winter circulation J Geophys Res-Oceans 119:2263-2289 doi:Doi 10.1002/2013jc009331

Yao FC, Hoteit I, Pratt LJ, Bower AS, Zhai P, Kohl A, Gopalakrishnan G (2014b) Seasonal overturning circulation in the Red Sea: 1 . Model validation and summer circulation J Geophys Res-Oceans 119:2238-2262 doi:Doi 10.1002/2013jc009004

Zhai P, Bower A (2013) The response of the Red Sea to a strong wind jet near the Tokar Gap in summer J Geophys Res-Oceans 118:23 doi:10.1029/2012JC008444

Zhai P, Pratt LJ, Bower A (2015) On the Crossover of Boundary Currents in an Idealized Model of the Red Sea J Phys Oceanogr 45:1410-1425 
Zhai XM, Marshall DP (2013) Vertical Eddy Energy Fluxes in the North Atlantic Subtropical and Subpolar Gyres J Phys Oceanogr 43:95-103 doi:10.1175/JpoD-12-021.1

Zhan P, Subramanian AC, Yao FC, Hoteit I (2014) Eddies in the Red Sea: A statistical and dynamical study J Geophys Res-Oceans 119:3909-3925

Zhan P, Subramanian AC, Yao FC, Kartadikaria AR, Guo D, Hoteit I (2016) The eddy kinetic energy budget in the Red Sea J Geophys Res-Oceans Accepted Author Manuscript. doi:10.1002/2015JC011589 


\section{List of figures}

Fig. 1 Mean and standard deviation of remote sensing observations (upper panel) and model outputs (lower panel) calculated using data from 1996 to 2010.

Fig. 2 DART-MITgcm EAKF scheme flow chart. The forecast ensemble $X^{\wedge} f$ is first updated with DART using the observation to compute the new analysis ensemble $\mathrm{X}^{\wedge} \mathrm{a}$. The latter is then integrated with the MITgcm to obtain the forecast ensemble $\mathrm{X}^{\wedge} \mathrm{f}$ from which a new assimilation cycle could be initiated.

Fig. 3 DART-MITgcm EnOI scheme flow chart. The forecast ensemble $X^{\wedge} f$ is first updated with DART based on the observation to compute the new analysis ensemble mean $\mathrm{x}^{\wedge} \mathrm{a}$, which is then integrated with the MITgcm to obtain the forecast $\mathrm{x}^{\wedge} \mathrm{f}$. The single forecast is added to a pre-selected ensemble of anomalies to build the forecast ensemble $X^{\wedge} f=x^{\wedge} f+X^{\prime}$, from which a new assimilation cycle could be initiated.

Fig. 4 Time evolution of the SST/SSH RMSE and ensemble spread for EAKF with different ensemble sizes.

Fig. 5 Time evolution of the SST/SSH RMSE and ensemble spread for EAKF with different inflation factors.

Fig. 6 Time evolution of the SST/SSH RMSE and ensemble spread for EAKF (ensemble size $=100)$, EnOI (ensemble size $=250)$ and SEnOI (ensemble size of 250).

Fig. 7 (a)-(g): SSH forecast/analysis from EAKF, EnOI and SEnOI compared with gridded AVISO product on June-6-2006, superimposed with the along-track altimeter data. (h)-(j) SSH increment from EAKF, EnOI and SEnOI. 
Fig. 8 (a)-(g): SST forecast/analysis from EAKF, EnOI and SEnOI compared with AVHRR product on June-6-2006. (h)-(m): Vertical structure along a cross section of the Red Sea axis plotted as the black line in (a).

Fig. 9 Horizontal and vertical distributions of ensemble spread (a)-(c) of SSH, (d)-(f) SST, and (g)-(i) temperature on June-6-2006 as they result from the EAKF, EnOI and SEnOI.

Fig. 10 (a)-(f) Zonal and meridional residue terms calculated from the analysis fields of EAKF, EnOI and SEnOI on June-6-2006. (g)-(l) Comparison of zonal and meridional Coriolis term and horizontal pressure gradient term for EAKF, EnOI and SEnOI along the Red Sea axis (plotted as the black line in (a)) on June-6-2006.

Fig. 11 Time evolution of the SST/SSH RMSE for EAKF ((a) to (d)) and a free-run simulation ((e) and (f)), both forced with ECMWF and NCEP.

Fig. 12 Horizontal and vertical distribution of forecast temperature averaged in August (a)-(h) and in December (i)-(p). The left/right two panels plot the forecast fields from an EAKF/free run experiment, forced with ECMWF and NCEP atmospheric conditions, respectively. 
Figures
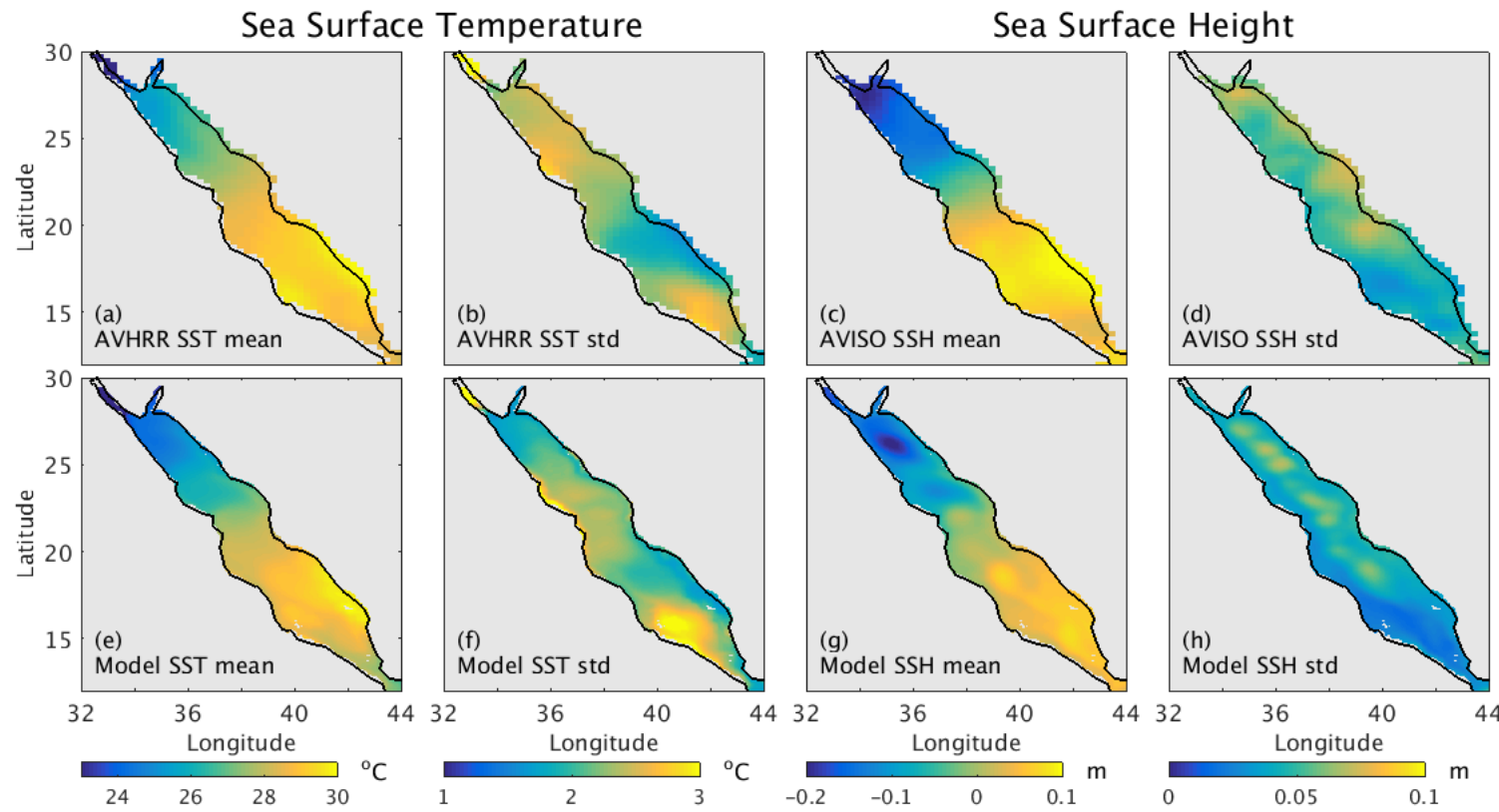

Fig. 1 Mean and standard deviation of remote sensing observations (upper panel) and model outputs (lower panel) calculated using data from 1996 to 2010. 


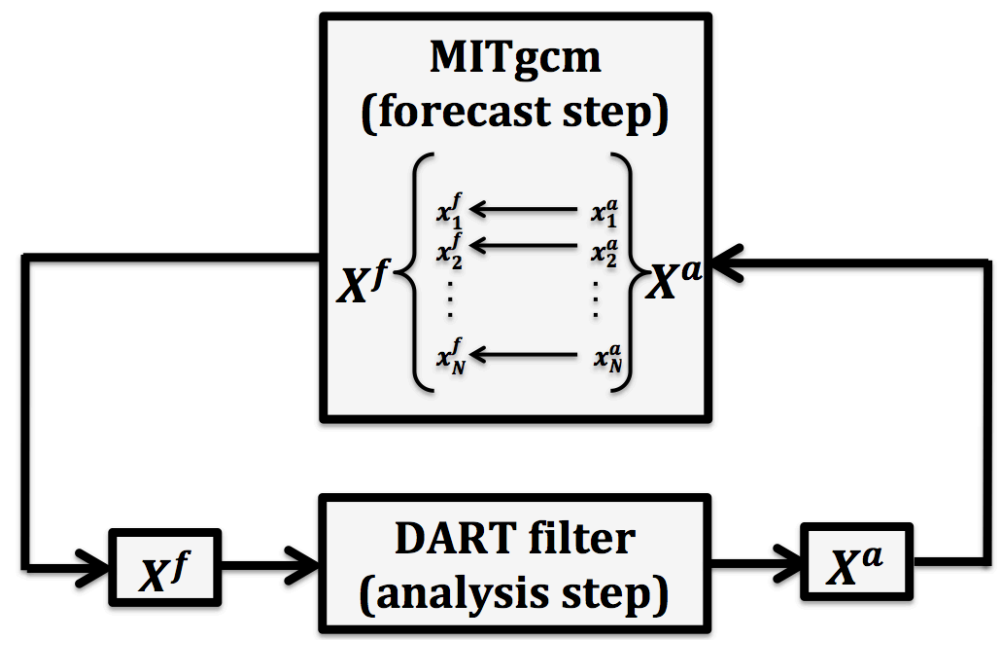

Fig. 2 DART-MITgcm EAKF scheme flow chart. The forecast ensemble $X^{f}$ is first updated with DART using the observation to compute the new analysis ensemble $X^{a}$. The latter is then integrated with the MITgcm to obtain the forecast ensemble $X^{f}$ from which a new assimilation cycle could be initiated. 


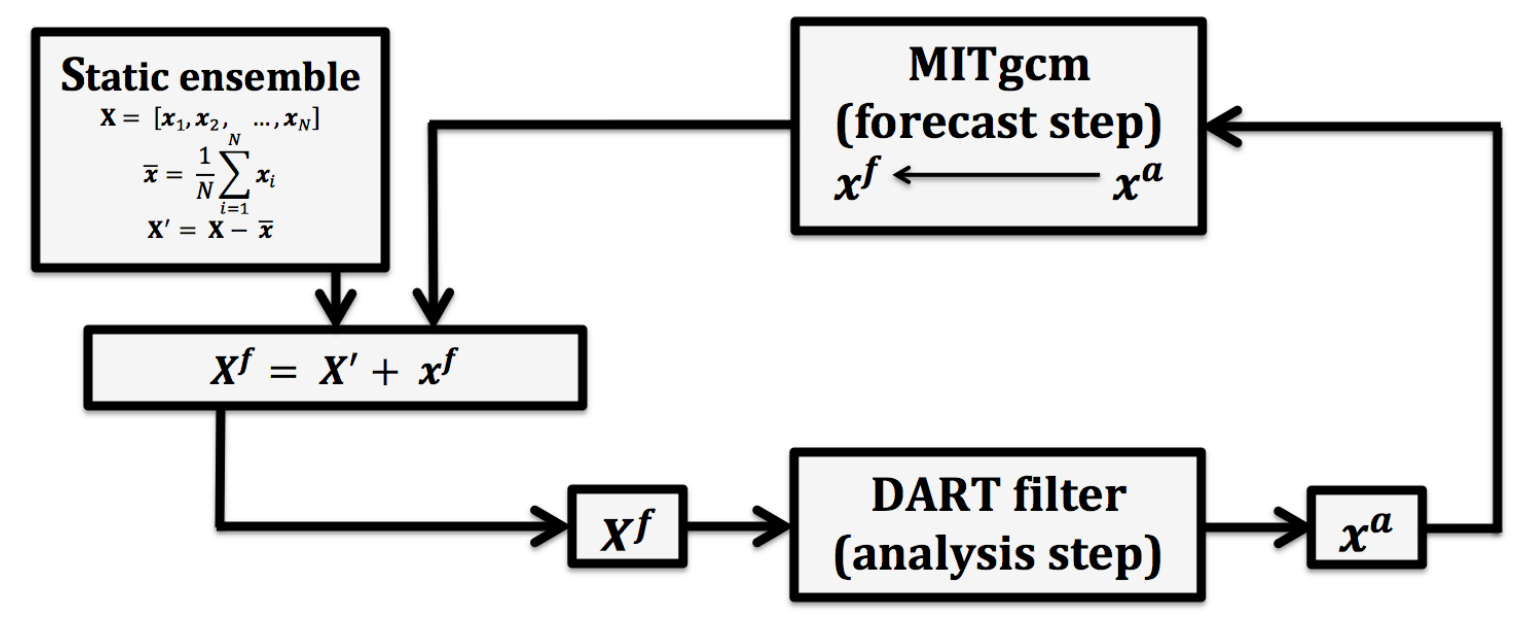

Fig. 3 DART-MITgcm EnOI scheme flow chart. The forecast ensemble $X^{f}$ is first updated with DART based on the observation to compute the new analysis ensemble mean $x^{a}$, which is then integrated with the MITgcm to obtain the forecast $x^{f}$. The single forecast is added to a pre-selected ensemble of anomalies to build the forecast ensemble $X^{f}=x^{f}+X^{\prime}$, from which a new assimilation cycle could be initiated. 

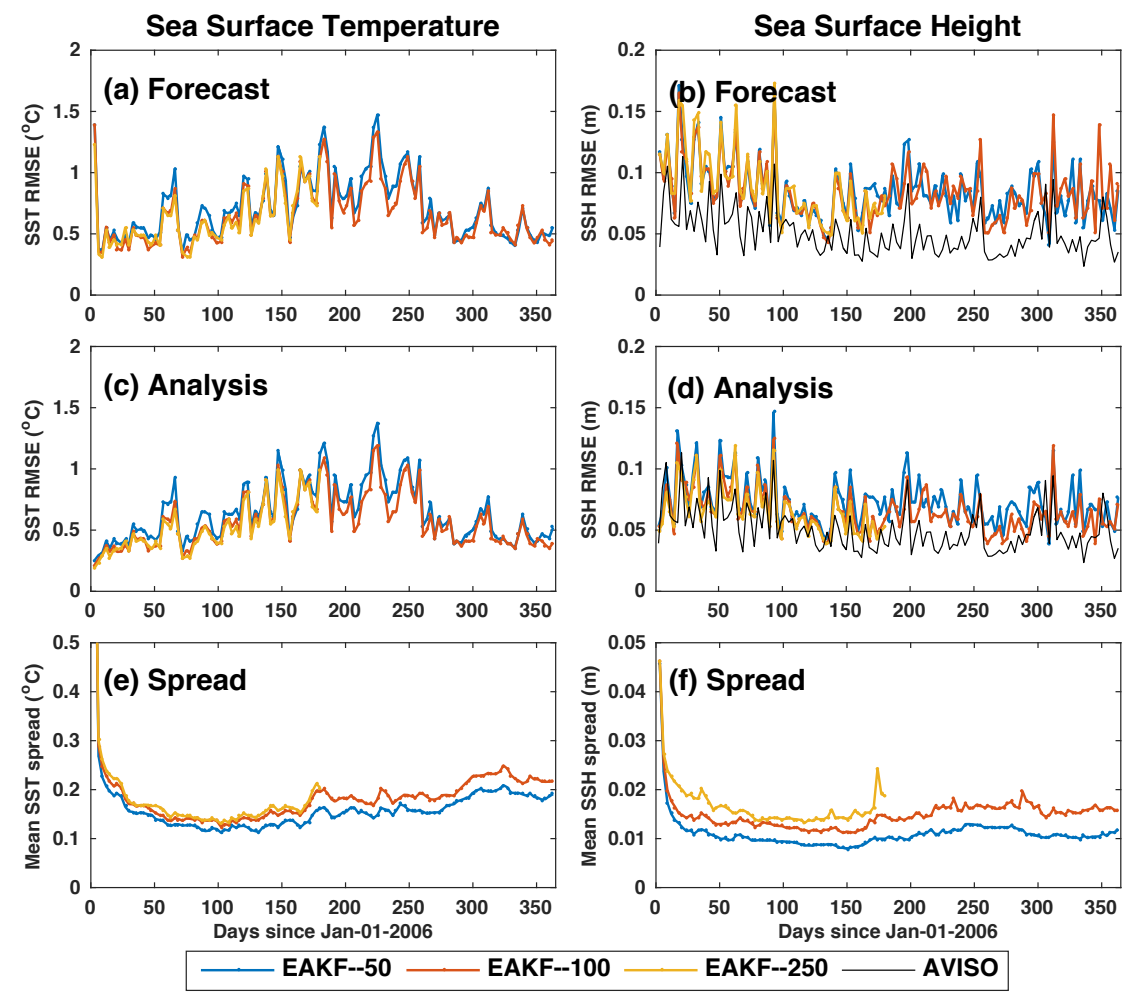

Fig. 4 Time evolution of the SST/SSH RMSE and ensemble spread for EAKF with different ensemble sizes. 

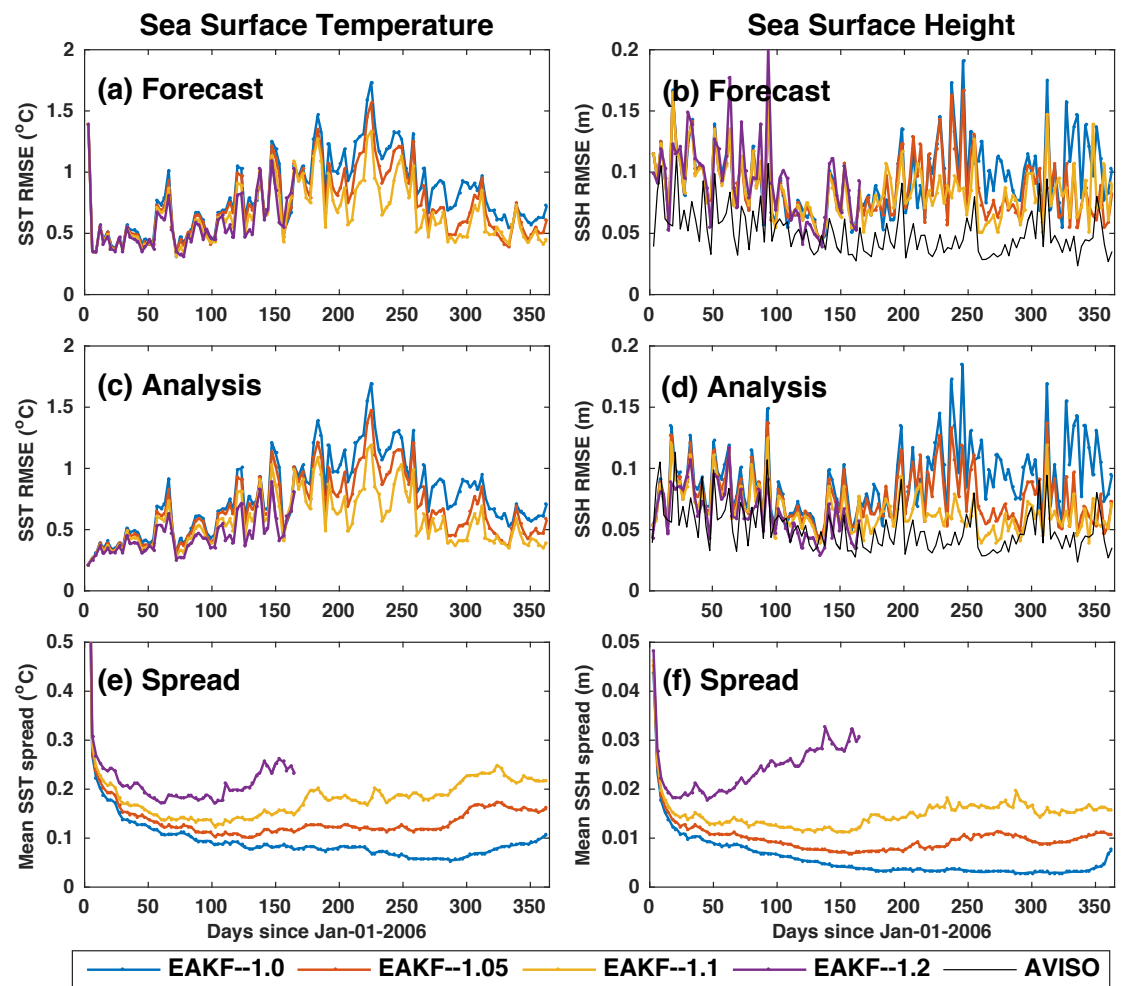

Fig. 5 Time evolution of the SST/SSH RMSE and ensemble spread for EAKF with different inflation factors. 

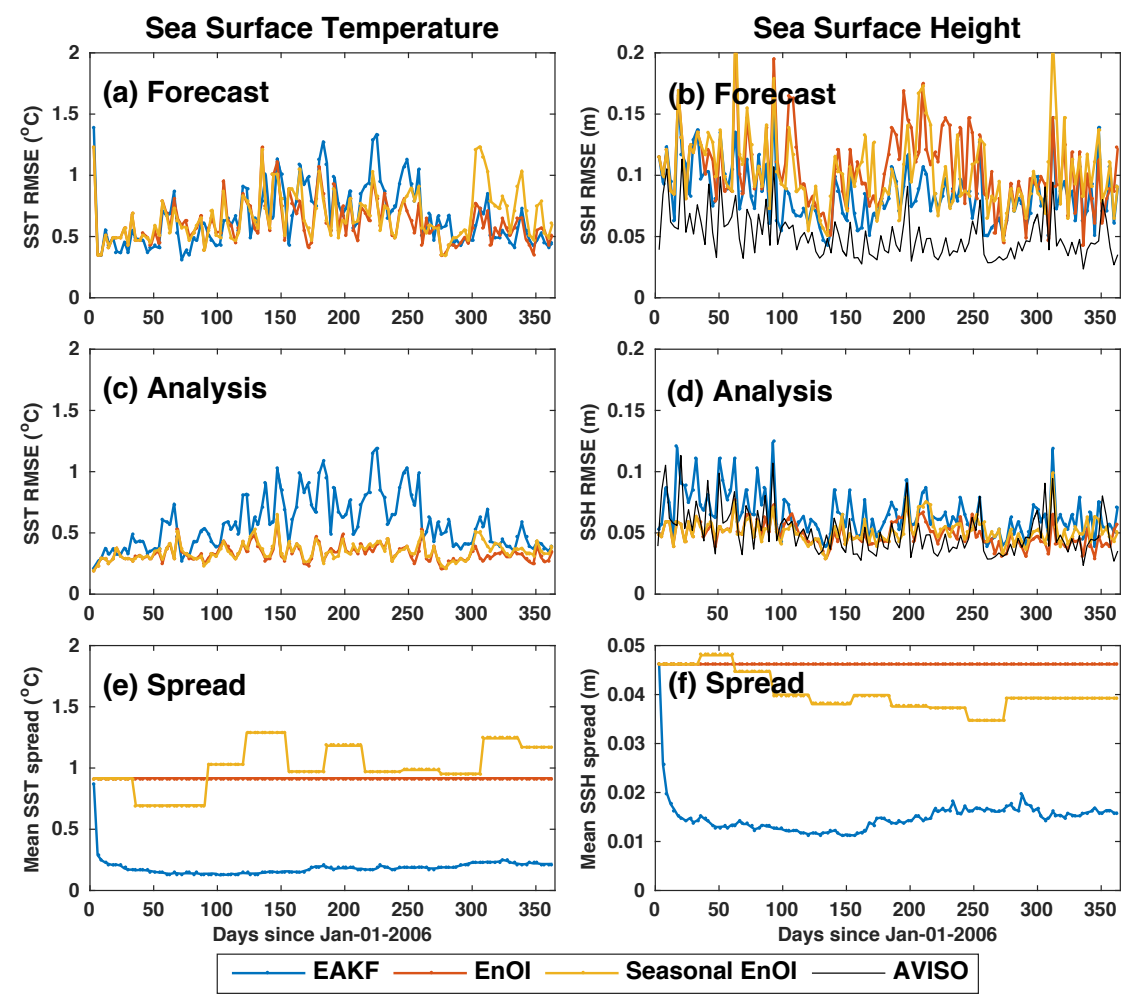

Fig. 6 Time evolution of the SST/SSH RMSE and ensemble spread for EAKF (ensemble size $=100)$, EnOI (ensemble size $=250)$ and SEnOI (ensemble size of 250). 

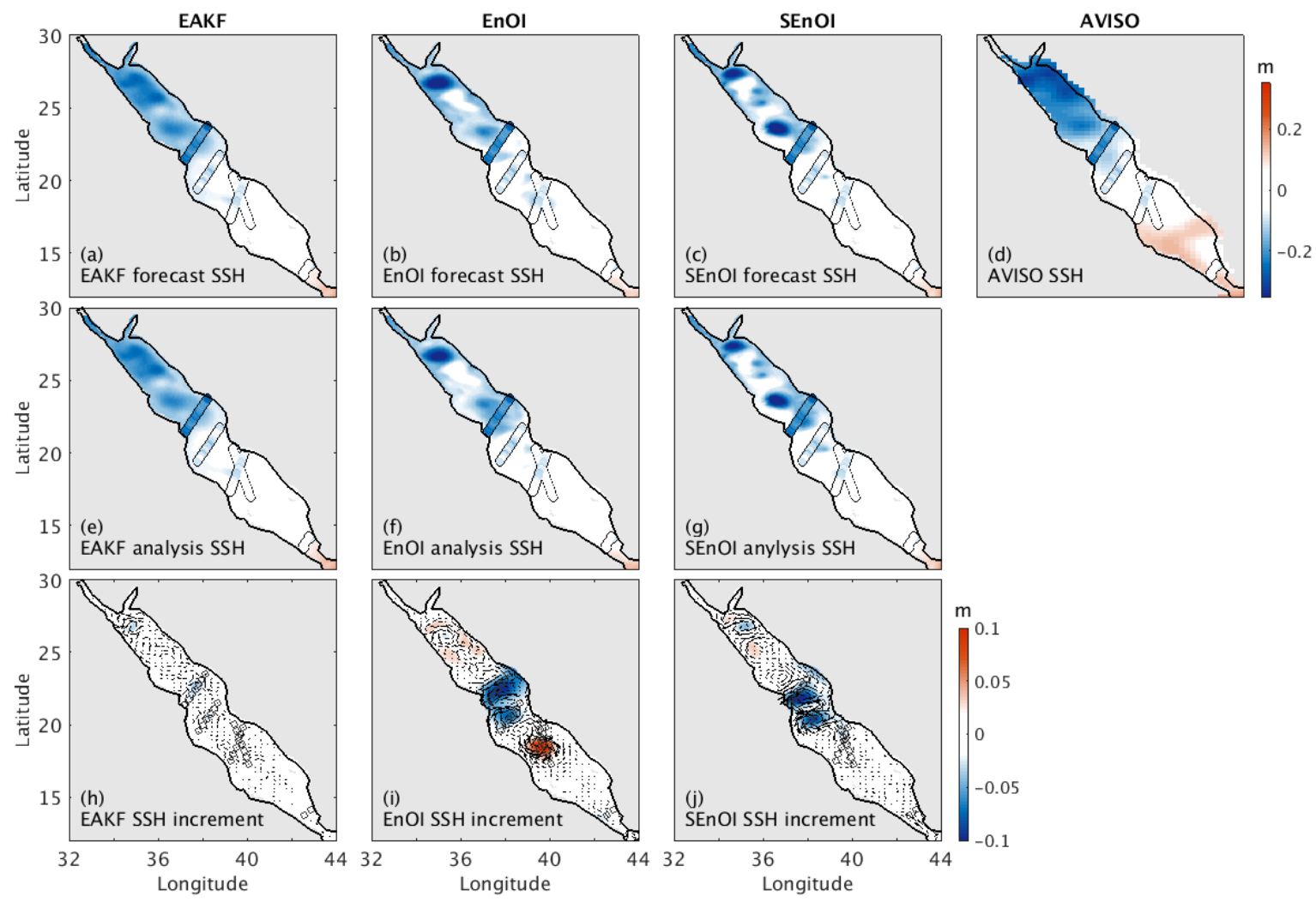

Fig. 7 (a)-(g): SSH forecast/analysis from EAKF, EnOI and SEnOI compared with gridded AVISO product on June-6-2006, superimposed with the along-track altimeter data. (h)-(j) SSH increment from EAKF, EnOI and SEnOI. 

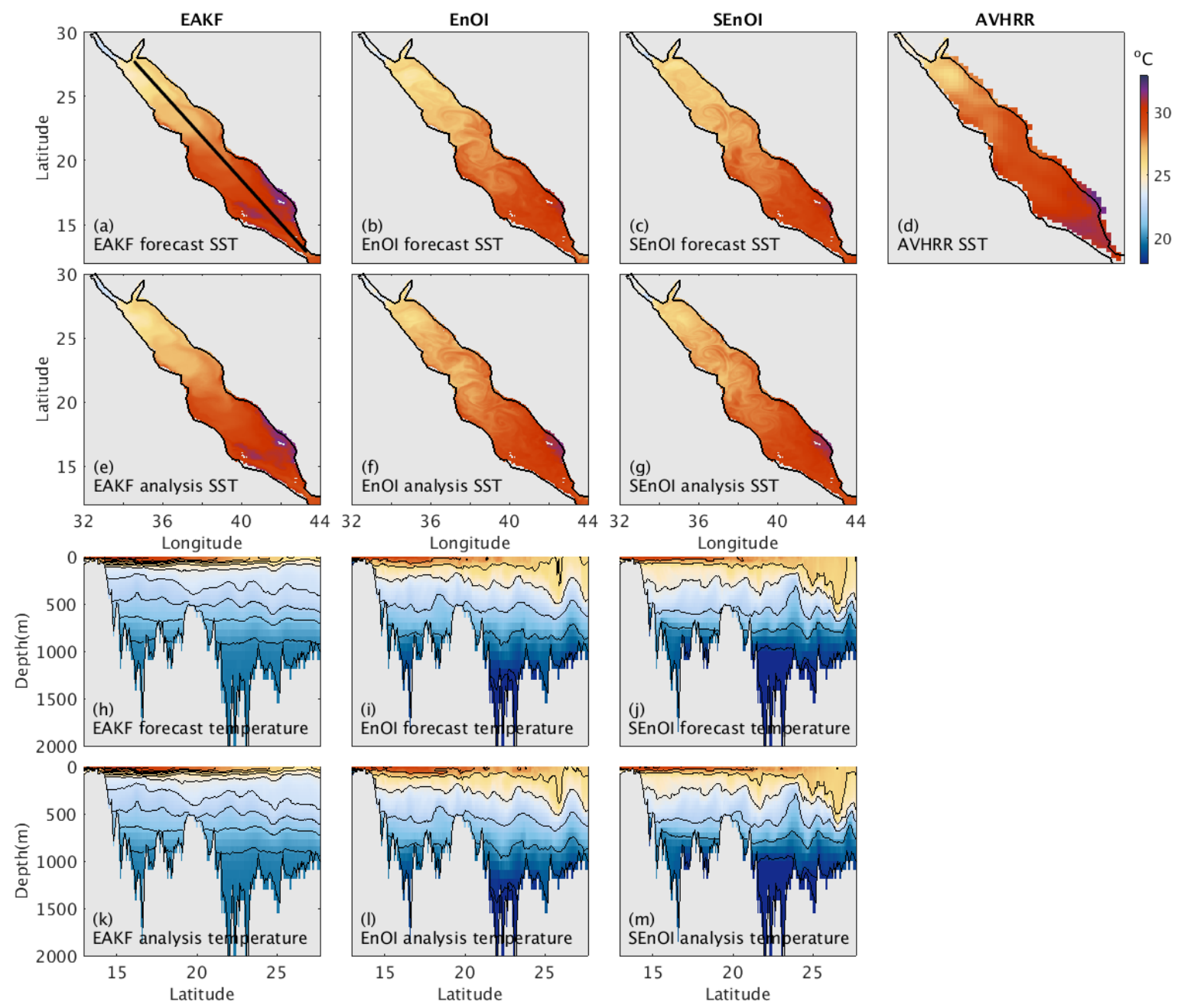

Fig. 8 (a)-(g): SST forecast/analysis from EAKF, EnOI and SEnOI compared with AVHRR product on June-6-2006. (h)-(m): Vertical structure along a cross section of the Red Sea axis plotted as the black line in (a). 

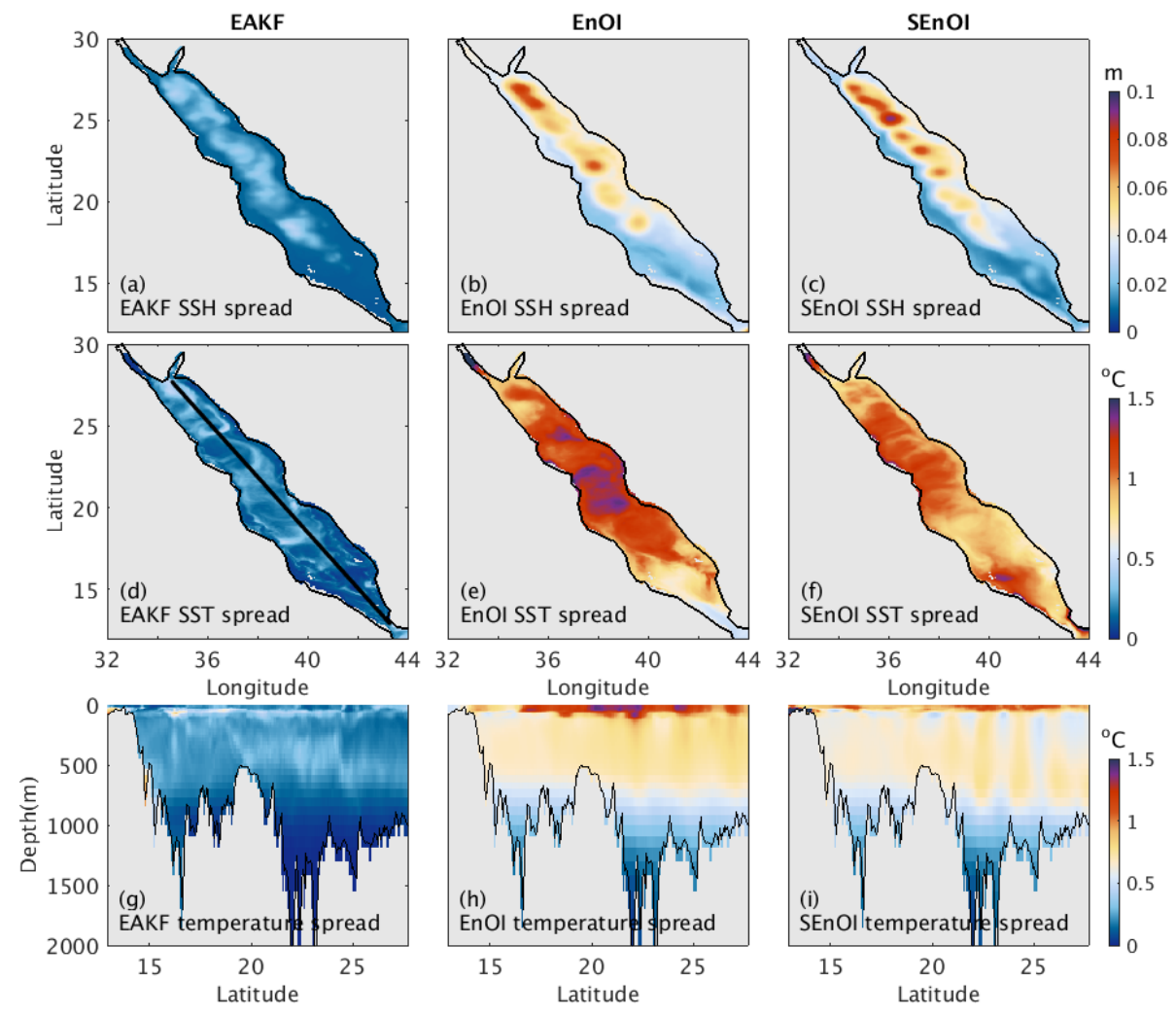

Fig. 9 Horizontal and vertical distributions of ensemble spread (a)-(c) of SSH, (d)-(f) SST, and (g)-(i) temperature on June-6-2006 as they result from the EAKF, EnOI and SEnOI. 

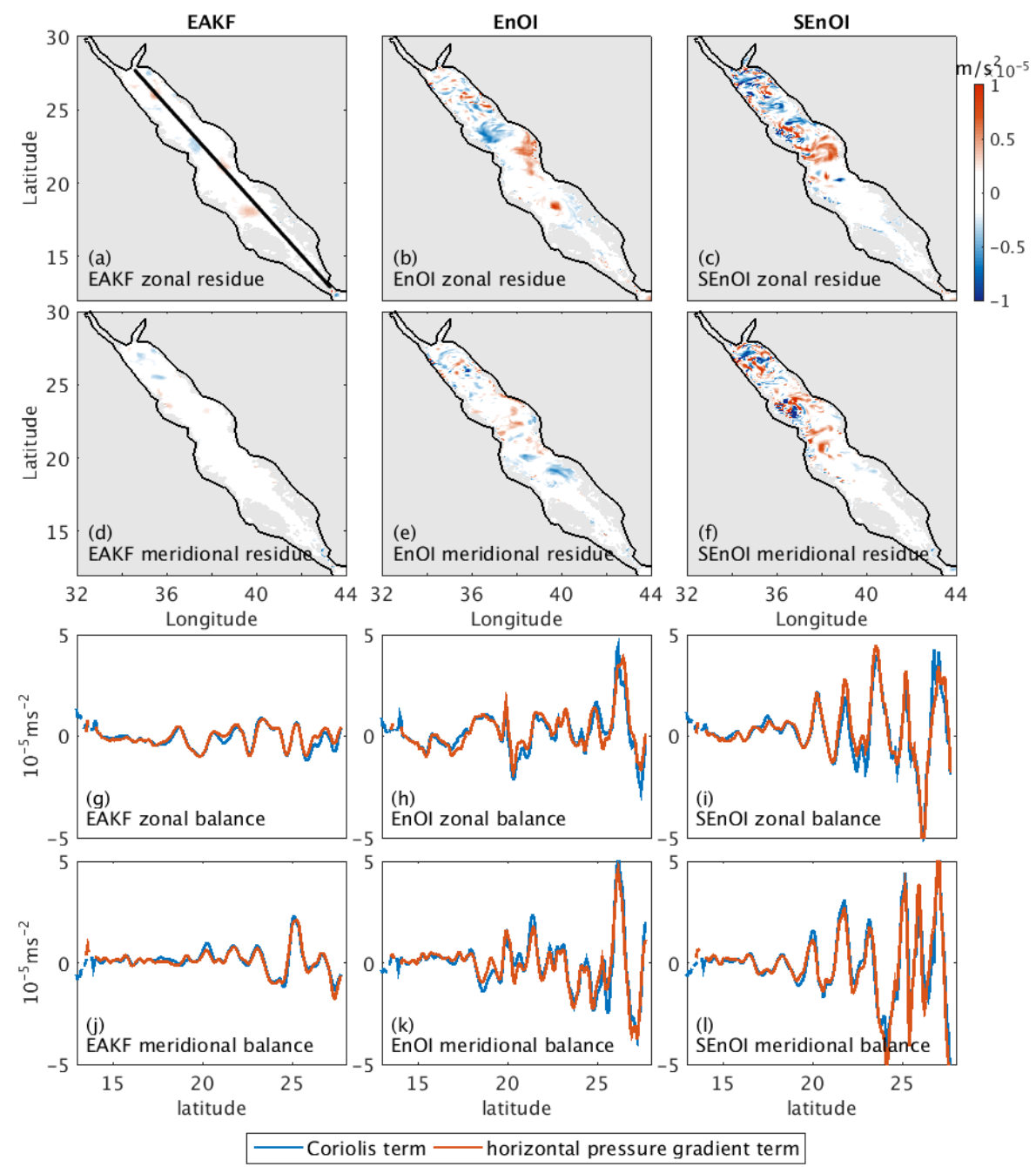

Fig. 10 (a)-(f) Zonal and meridional residue terms calculated from the analysis fields of EAKF, EnOI and SEnOI on June-6-2006. (g)-(1) Comparison of zonal and meridional Coriolis term and horizontal pressure gradient term for EAKF, EnOI and SEnOI along the Red Sea axis (plotted as the black line in (a)) on June-6-2006. 

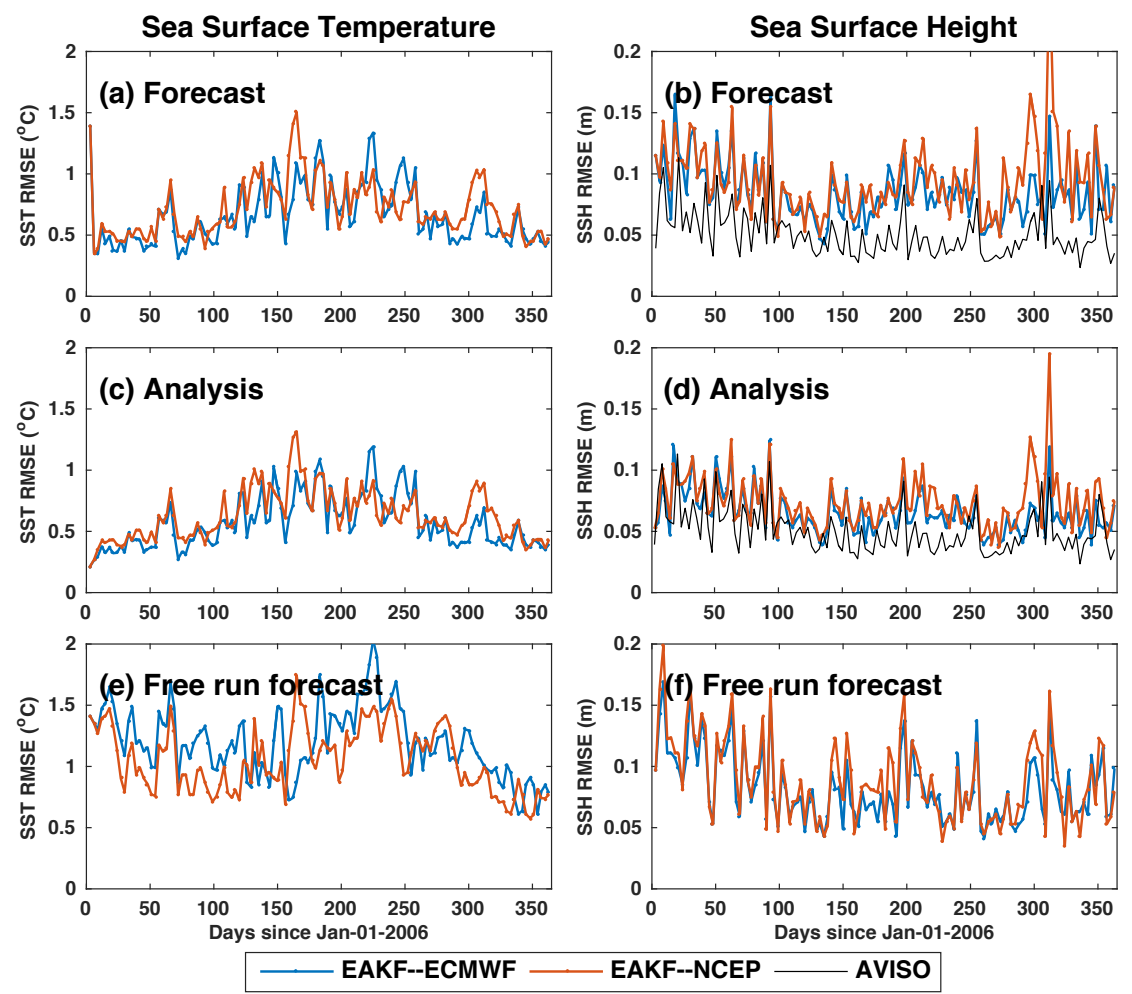

Fig. 11 Time evolution of the SST/SSH RMSE for EAKF ((a) to (d)) and a free-run simulation ((e) and (f)), both forced with ECMWF and NCEP. 

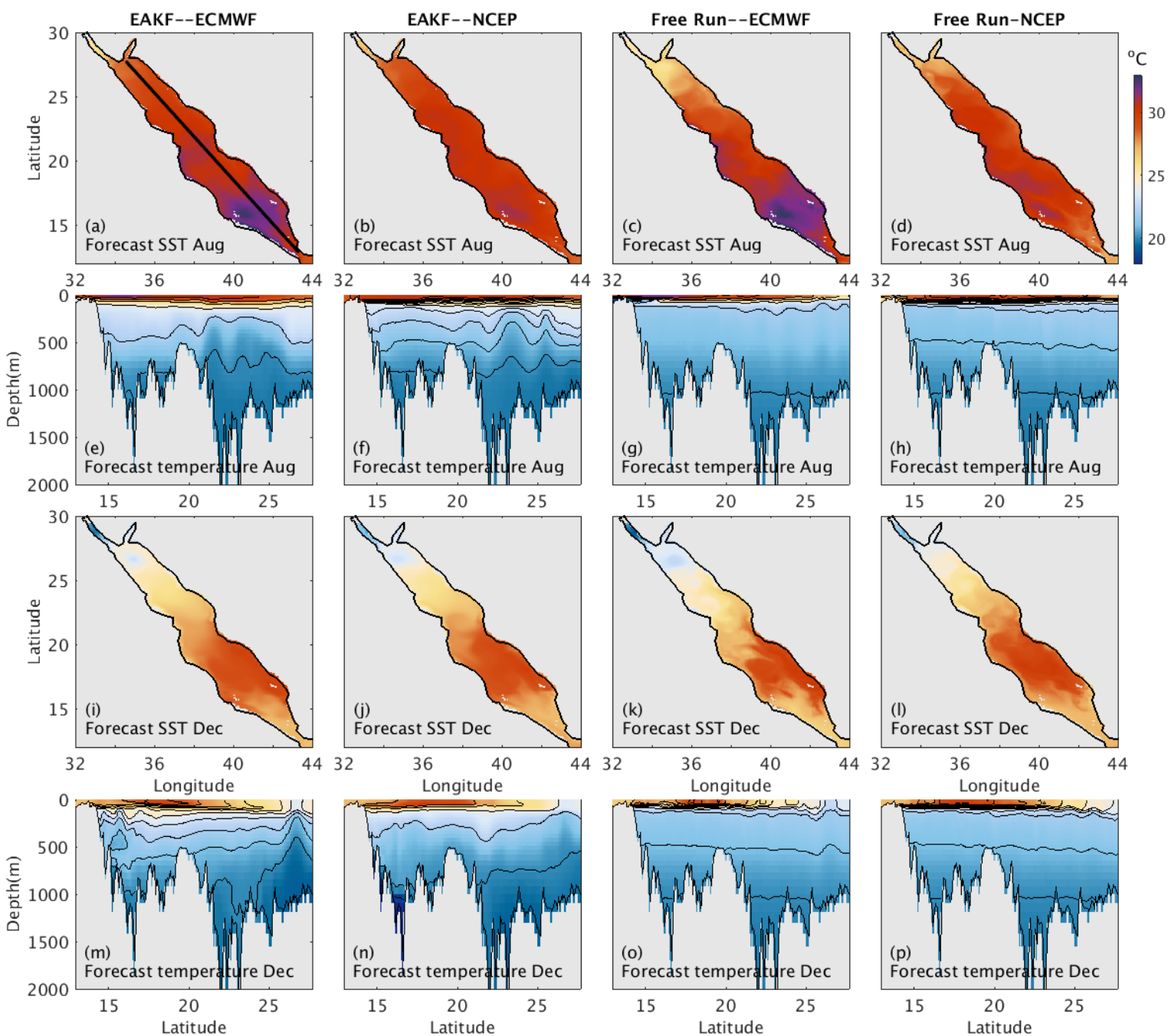

Fig. 12 Horizontal and vertical distribution of forecast temperature averaged in August (a)-(h) and in December (i)-(p). The left/right two panels plot the forecast fields from an EAKF/free run experiment, forced with ECMWF and NCEP atmospheric conditions, respectively. 